\title{
Binocular unmasking with unequal interocular contrast: The case for multiple Cyclopean eyes
}

\author{
BRUCE SCHNEIDER and GIAMPAOLO MORAGLIA \\ University of Toronto, Erindale Campus, Mississauga, Ontario, Canada
}

\begin{abstract}
Under certain conditions, the detection threshold for a sinusoidal grating embedded in a noisy background may be an order of magnitude lower when binocular cues are available than when monocular cues only are present. Such binocular unmasking occurs only when the degree of interocular disparity for the target differs from that of the background. Two classes of models have been advanced to account for such unmasking. The first assumes that orientation-specific, spatial frequency channels in each eye encode the amplitude and phase of the spatial frequency component of the pattern the channel is tuned to detect. Thus, a difference in interocular disparity between target and background could result in interocular amplitude and/or phase differences in left- and right-eye spatial frequency channels. When, however, there are no disparity differences between target and background, there will be no interocular differences in amplitude and phase in the left- and right-eye channels. In this model, then, binocular unmasking reflects the binocular system's ability to respond to interocular amplitude and/or phase differences in the patterns presented to the two eyes. In the second class of models, it is assumed that the left- and right-eye patterns are first summed to form a "Cyclopean" eye. In these models, detection depends on the effect this summation process has on the power spectrum of the summated patterns. To decide between these two classes of models, we observed the occurrence of binocular unmasking when (1) the contrast of masker and signal was varied identically in both eyes and (2) the contrast of masker and signal was varied in one eye only. Consistent with our previous research, we found that the results can be accounted for in terms of a linear summation model of binocular unmasking; the alternative interocular phase detection model was disproved. The implications of these findings for binocular contrast summation in the absence of visual noise are discussed.
\end{abstract}

The detection threshold for a sinusoidal grating embedded in a noisy background may be more than an order of magnitude lower when binocular cues are available than when only monocular cues are present (Henning \& Hertz, 1973, 1977; Moraglia \& Schneider, 1990, 1991, 1992; Schneider, Moraglia, \& Jepson, 1989). This effect, in deference to its auditory counterpart (see Durlach \& Colburn, 1978, for a review), has been termed binocular unmasking. We (Moraglia \& Schneider, 1990, 1991, 1992; Schneider et al., 1989) have suggested that binocular unmasking may result from a linear summation of monocular inputs, through the effects that this operation has on the power spectrum of the summated input and thus on the resulting signal-to-noise ratio in the summated pattern. Alternatively, other authors, both in audition (Jeffress, 1972; McFadden, 1968) and in vision (Henning \& Hertz, 1973, 1977) have proposed that the unmasking effect can be attributed to the observer's ability to dis-

This research was supported by grants from the Natural Sciences and Engineering Research Council of Canada to each author. We thank Scott Parker for his comments on an earlier version of this manuscript. Requests for reprints should be sent to B. Schneider, Department of Psychology, University of Toronto, Erindale Campus, Mississauga, ON L5L 1C6, Canada. criminate interaural or interocular phase differences. In these models, vector diagrams are constructed for noise alone and signal + noise trials for each ear or eye. It is then assumed that the observer uses interaural or interocular differences in phase to detect the presence of the signal. In the auditory realm, where the predictions of these competing views have been explored most thoroughly, it is typically found that both models, with appropriate modifications, can account equally well for most phenomena (see Colburn \& Durlach, 1978, for a review). In the visual realm, it is also clear that the vector model and the summation model can both readily account for the effects so far investigated (Henning \& Hertz, 1973, 1977; Moraglia \& Schneider, 1990, 1991, 1992; Schneider et al., 1989).

In order to overcome this theoretical impasse, in the present experiments we resorted to conditions of stimulation in which vector models and linear summation models predict quite different degrees of unmasking. In particular, binocular unmasking was determined when (1) the root-mean-square (RMS) contrasts of both masker and signal were equal in both eyes and when (2) the RMS contrasts of both masker and signal in one eye were either $50 \%$ or $25 \%$ of the value in the other eye. To explicate why the two models make different predictions, we will first describe the experimental paradigm employed in our 
studies and then show how the two models account for the occurrence of binocular unmasking in these conditions.

\section{The Experimental Paradigm}

As in our previous research, we employed here a standard two-interval, forced-choice paradigm, in which ob- servers were presented with two image pairs viewed through a simple-lens stereoscope. On noise-alone intervals, a field of two-dimensional (2-D) broadband Gaussian noise $(N)$ surrounded by a square frame of uniform noise was presented to each eye (see middle panel of Figure 1). The 2-D noise field presented to the right eye,
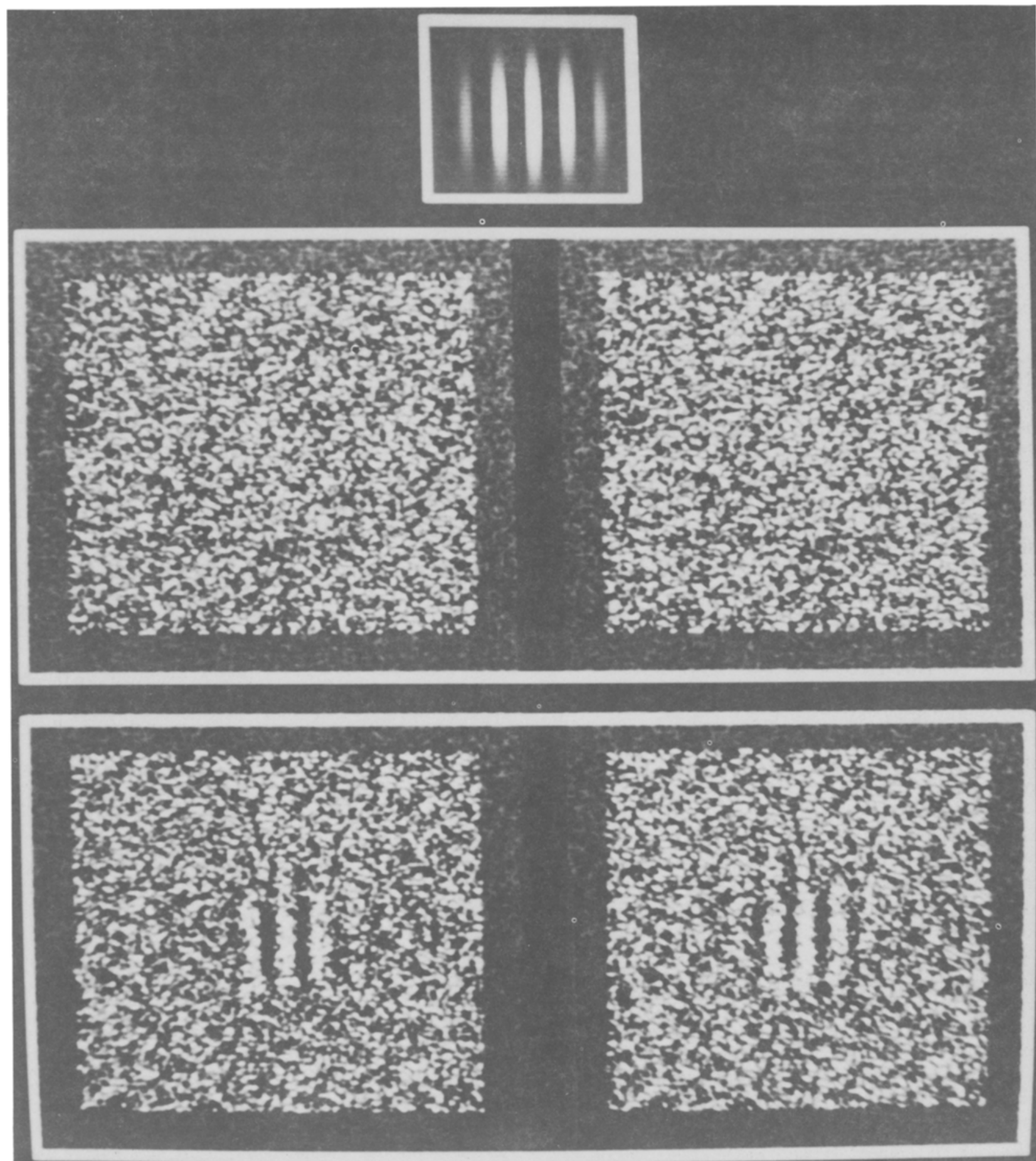

Figure 1. The upper panel shows the Gabor signal used in all experiments. The middle panel shows a noise-only display; relative to the left one, the right-eye Gaussian noise field is shifted horizontally to the right by 6 pixels $\left(20.3^{\prime}\right.$ of arc). The lower panel shows the Gabor patch embedded in the noise, as in the experimental $N_{d} S_{0}$ condition. 
however, was shifted $d_{x}$ to the right within the surrounding frame, as with uncrossed disparity. When viewed through the stereoscope the fused 2-D noise was therefore perceived as being located behind the surrounding frame. Before each trial began, a fixation spot was presented in the center of each frame. When the observer fixated on this spot, this point, $\left(x_{0}, y_{0}\right)$, was thus imaged in the center of each fovea. Let the luminance pattern for the left eye be $g(x, y)$. Since the right-eye pattern was shifted $d_{x}$ to the right (shifts to the right are given a negative sign), the luminance pattern for the right eye is then given by $g\left(x+d_{x}, y\right)$. On signal + noise intervals, a Gaussian-enveloped sine-wave grating of the Gabor type (Daugman, 1980), whose wavelength was equal to $2 d_{x}$ (see top panel of Figure 1), was added to each field (see bottom panel of Figure 1).

On the set of trials that were designed to produce binocular unmasking, the Gabor signal, which was here oriented vertically, was added at the center of both the left- and the right-eye noise fields. Thus, with reference to the frames, the coordinates of the Gabor patterns were identical in both eyes $\left(N_{d} S_{0}\right.$ condition). When viewed through the stereoscope, the fused signal appeared to float in front of the background noise on the same plane as that of the frame, which also had identical coordinates in both eyes.

On control trials, the Gabor signal presented to the right eye was shifted $d_{x}$ to the right of center. Since the 2-D noise background and the Gabor signal were shifted by the same amount $\left(N_{d} S_{d}\right.$ condition), the signal, when viewed through the stereoscope, appeared to be embedded within the background noise located behind the frame. The degree of unmasking is indexed by the decibel difference in threshold signal-to-noise ratio in the two conditions and is referred to as a binocular masking-level difference (BMLD). The BMLD, measured in decibels, equals $20 \log \left[T\left(N_{d} S_{d}\right) / T\left(N_{d} S_{0}\right)\right]$, where $T\left(N_{d} S_{d}\right)$ and $T\left(N_{d} S_{0}\right)$ represent the RMS contrasts at threshold for Conditions $N_{d} S_{d}$ and $N_{d} S_{0}$, respectively. BMLDs found in various experiments range from 6 to $20 \mathrm{~dB}$.

\section{The Summation Model}

We propose, in the summation model, that the monocular inputs to the two eyes are added together to produce a Cyclopean visual field (Julesz, 1971); of course, before addition occurs, the two eyes must be aligned through appropriate vergence movements. In our experiment, this alignment is prompted by the presence of a fixation point. We also propose that summation occurs not only for corresponding points on the retinas, but also for points shifted horizontally or vertically. How this might occur is shown in Figure 2. The circles depict points on the two retinas; the circles that have the same retinal coordinate values thus stand for corresponding points on the two retinas. Let us assume that corresponding points are simply added to form one Cyclopean eye. It is also possible that a second, parallel Cyclopean eye is formed by adding point $(x, y)$ in the left eye to point $\left(x+d_{\alpha}, y\right)$ in the right eye, where $d_{\alpha}$ represents either a positive or a negative hori-



Figure 2. Upper panel: Retinal points in the keft and right eye are indicated by circles whose horizontal and vertical positions, relative to the center of the fovea, are specified by the horizontal and vertical axes. Lower panel: For one row of left- and right-eye points, the left- and right-eye inputs to the Cyclopean eye are defined by $\left(d_{\mathrm{a}}=-2, d_{\mathrm{s}}=0\right)$.

zontal shift. For example, if $d_{\alpha}=-2$, then point $(0,0)$ in the left eye would be added to point $(-2,0)$ in the right eye and so on for all retinal points. Other Cyclopean eyes could then be formed by adding any combination of horizontal or vertical shifts within a limited range. Thus, many different Cyclopean eyes could be realized by this form of parallel additive processing.

The net result would be that if luminance pattern $f(x, y)$ were presented to the left eye, and pattern $f\left(x+d_{x}, y+d_{y}\right)$ to the right eye, where $d_{x}$ and $d_{y}$ represent horizontal and vertical displacements in the stimulus pattern, the summated pattern in the Cyclopean eye defined by shifts $d_{\alpha}$ and $d_{\beta}$, where $\alpha$ refers to a horizontal shift and $\beta$ to a vertical shift, would be

$$
f(x, y)+f\left(x+d_{x}+d_{\alpha}, y+d_{y}+d_{\beta}\right) .
$$

Suppose that $f(x, y)=g(x, y)$, that is, $f(x, y)$ is a 2-D bandlimited Gaussian noise, $g(x, y)$, whose power spectral density function,

$$
\begin{aligned}
G(\epsilon, \eta)= & A_{g},-\epsilon_{0} \leq \epsilon \leq \epsilon_{0} \text { and }-\eta_{0} \leq \eta \leq \eta_{0} \\
& 0, \text { elsewhere, }
\end{aligned}
$$

with $A_{g}$ representing the spectrum level of the noise, $\epsilon$ and $\eta$ the spatial frequency variables corresponding to the horizontal and vertical axes, and $\epsilon_{0}$ and $\eta_{0}$ the upper horizontal and vertical frequency limits on the band-limited noise. Assume that $d_{\alpha}=d_{\beta}=d_{y}=0$, and that $d_{x} \neq 0$. Formula 1 now becomes

$$
g(x, y)+g\left(x+d_{x}, y\right),
$$

and its 2-D power spectral density function (see Moraglia 
\& Schneider, 1991) is

$$
G(\epsilon, \eta)\left[2+2 \cos \left(2 \pi \epsilon d_{x}\right)\right] \text {. }
$$

The summated $\left(d_{x}=20.3^{\prime}\right.$ of arc) 2-D spectral power density function for a Gaussian noise (bandwidth limited for illustrative purposes to $0-3$ cycles/degree [cpd] along both horizontal and vertical spatial frequency axes) is shown in Figure 3. Note that spectral power varies with horizontal spatial frequency only and that a minimum of zero occurs at $\epsilon=1 /\left(2 d_{\mathrm{x}}\right)=1.48 \mathrm{cpd}$. Therefore, vertically oriented, binocular spatial frequency channels, optimally tuned to $f=1.48 \mathrm{cpd}$, would not respond to the background noise. Consider, however, what would happen if a vertically oriented Gabor signal (spatial frequency $=1.48 \mathrm{cpd}$ ) were added without displacement to both visual fields. The luminance pattern of each Gabor is specified by

$j(x, y)=A_{j} \cos \left[2 \pi \epsilon_{s}\left(x-x_{0}\right)\right] \exp \left[-a^{2}\left(x-x_{0}\right)^{2}-a^{2}\left(y-y_{0}\right)^{2}\right]$,

where $A_{j}$ is the amplitude of the Gabor signal, $\epsilon_{s}$ its spatial frequency, $a$ the reciprocal of the space constant (one half the distance between the 1/e points on the Gaussian envelope), and $x_{0}, y_{0}$ the center of the enframed area. Because the Gabor signal is presented in exactly the same locations in the two visual fields, after summation by a Cyclopean eye whose $d_{\alpha}=d_{\beta}=0$, the summated pattern would simply be double that in Equation 5. The distribution of spectral power for the summated Gabor signal is shown in Figure 4 along with the spectral density function for the summated Gaussian noise. Clearly, a ver- tically oriented Gabor signal with spatial frequency equal to $1 /\left(2 d_{x}\right)$ should be readily visible against a Gaussian background where the horizontal shift in the right-eye background is equal to $d_{x}$.

Notice that, in this example, the power spectral density function for the summated noise is identical for all values of $\eta$; thus, it suffices for the present purposes to plot the spectral density function for the horizontal spatial frequency variable only. Figure 5 shows how the spectral density function for summated noise fields $\left(d_{x}=\right.$ $20.3^{\prime} ; d_{\alpha}, d_{\beta}=0$ ) varies as a function of the horizontal spatial frequency variable for the 2-D band-limited noise employed here (the full $0-8.7 \mathrm{cpd}$ noise bandwidth is shown). Note that there are troughs in the noise spectrum that occur at odd multiples of $1 /\left(2 d_{x}\right)$ and peaks that occur at even multiples of $1 /\left(2 d_{x}\right)$. To intuitively appreciate why the spectrum of $g(x, y)+g\left(x+d_{x}, y\right)$ is notched along the horizontal spatial frequency axis, recall that a band-limited Gaussian noise can be considered as a band of sinusoidal spatial frequencies whose amplitudes, phases, and orientations are random. Consider now the horizontal sine-wave grating with a wavelength equal to $2 d_{x}$ in $g(x, y)$. In $g\left(x+d_{x}, y\right)$, this sine-wave grating has been shifted by exactly one half of its wavelength, so that it is $180^{\circ}$ out of phase in the left- and right-eye patterns. Therefore, when the two patterns are added together in the Cyclopean eye, this sine-wave grating will be completely canceled. Wavelengths near $2 d_{x}$ will produce incomplete cancellation, resulting in the trough shown in Figure 5 at a frequency $=1 /\left(2 d_{x}\right)=1.48 \mathrm{cpd}$. On the other hand, a horizontal grating whose wavelength is $d_{x}$ will be $360^{\circ}$ out of phase and will show complete sum-

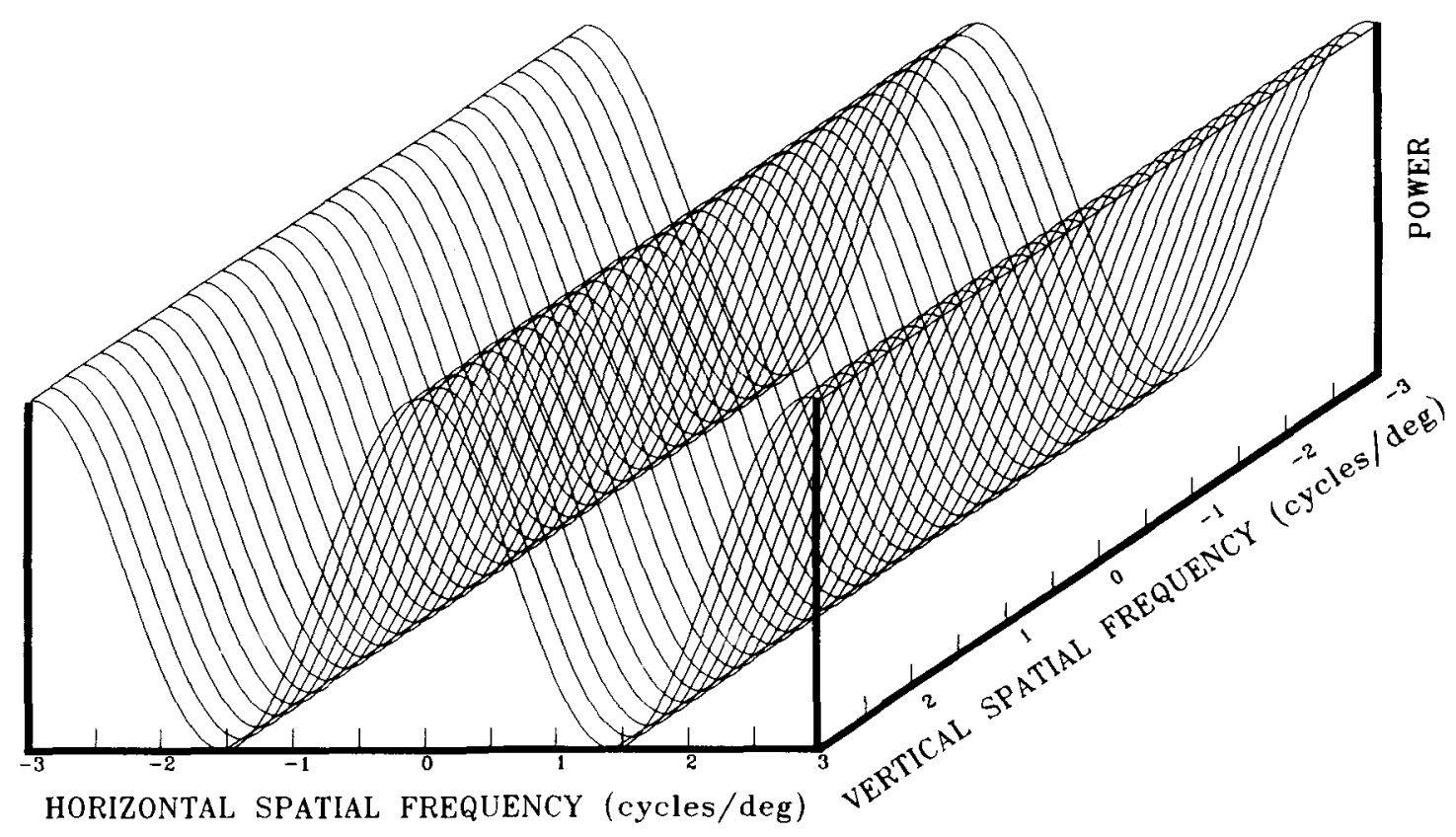

Figure 3. Two-dimensional power density function of the noise which results from the summation $\left(d_{\alpha}=d_{\beta}=0\right)$ of the Gaussian noises used in the experiments. Note that only the portion of the spectrum between \pm 3 cpd is plotted for purposes of clarity. 


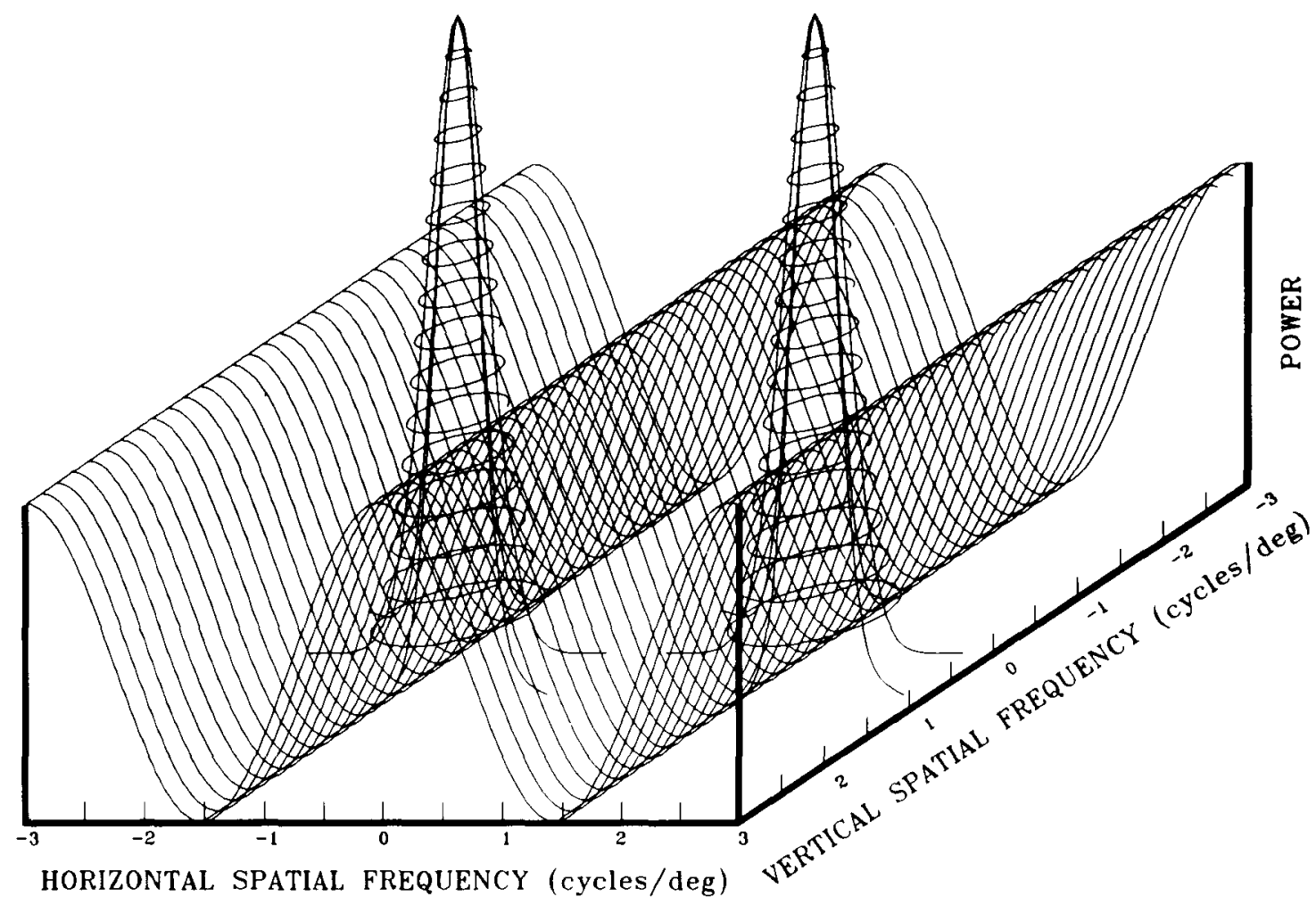

Figure 4. The two-dimensional power density function of the summated Gabor signal $\left(d_{\alpha}=d_{a}=0\right)$ is shown together with the corresponding function of the summated noise.

mation, producing a peak in the spectral density function at a frequency $1 / d_{x}=2.96 \mathrm{cpd}$.

Figure 5 also shows the horizontal profile of the Gabor signal; as the signal falls in a notch of the spectrum, it should be readily detected in the $N_{d} S_{0}$ condition. This opportunity, however, cannot be exploited in the $N_{d} S_{d}$ condition because the signals to be summated are in counterphase, since the displacement is exactly one half the wavelength of the signal. Therefore, in the latter condition, binocular summation essentially erases the signals in the Cyclopean eye. Because this condition contains no useful binocular cues, detection will then be based on information from the monocular channels only (e.g., Wolfe, 1986) or from the binocular channel (Cyclopean eye) with $d_{\alpha}=-d_{x}$ (see Appendix B).

Clearly, changing the displacements of the right- and left-eye noise patterns will change the 2-D spectral profile of the summated noise patterns. The 2-D spectral profile of both noise and signal can also be changed by changing the internally imposed shifts, $d_{\alpha}$ and $d_{\beta}$. Thus, different Cyclopean eyes are produced by different amounts of internally imposed disparity shifts. It is assumed in the model that the observer can attend to any one of these multiple Cyclopean eyes. Therefore, any combination of external or internal noise shifts that produces notches in the 2-D spectrum presents an opportunity for binocular unmasking, provided that the spatial frequency, orienta- tion, and degree of external interocular shift in the signal concentrate its energy at a notch of the summated 2-D noise spectrum for the Cyclopean eye in question. In every case so far investigated by us, and for the previous cases of BMLDs reported in the literature (Henning \& Hertz, 1973, 1977), binocular unmasking has occurred whenever the spectral energy in the summated signal was concentrated at a notch in the 2-D power spectrum of the summated noise for some reasonable values of $d_{\alpha}$ and $d_{\beta}$.

An examination of Figures 4 and 5 shows that, provided binocular spatial frequency channels are reasonably narrow, contrast thresholds for narrow-band Gabor signals should be virtually independent of noise level, because the 2-D power spectrum of the summated noise contains almost no energy in the region of the signal's frequency. Therefore, contrast thresholds for a Gabor signal in noise should be the same as those for a Gabor signal in the absence of noise; that is, unmasking should be nearly perfect. In order for the summation model to predict less than perfect unmasking, it is necessary to assume that there is internally generated random noise added to both the left- and right-eye patterns. For purposes of modeling, we assume that the bandwidths of these internally generated Gaussian noises are at least as large as the range of spatial frequencies that an observer is sensitive to; that is, the power spectral density functions for each of these noises, $h_{\mathrm{L}}(x, y)$ and $h_{\mathrm{R}}(x, y)$, are 


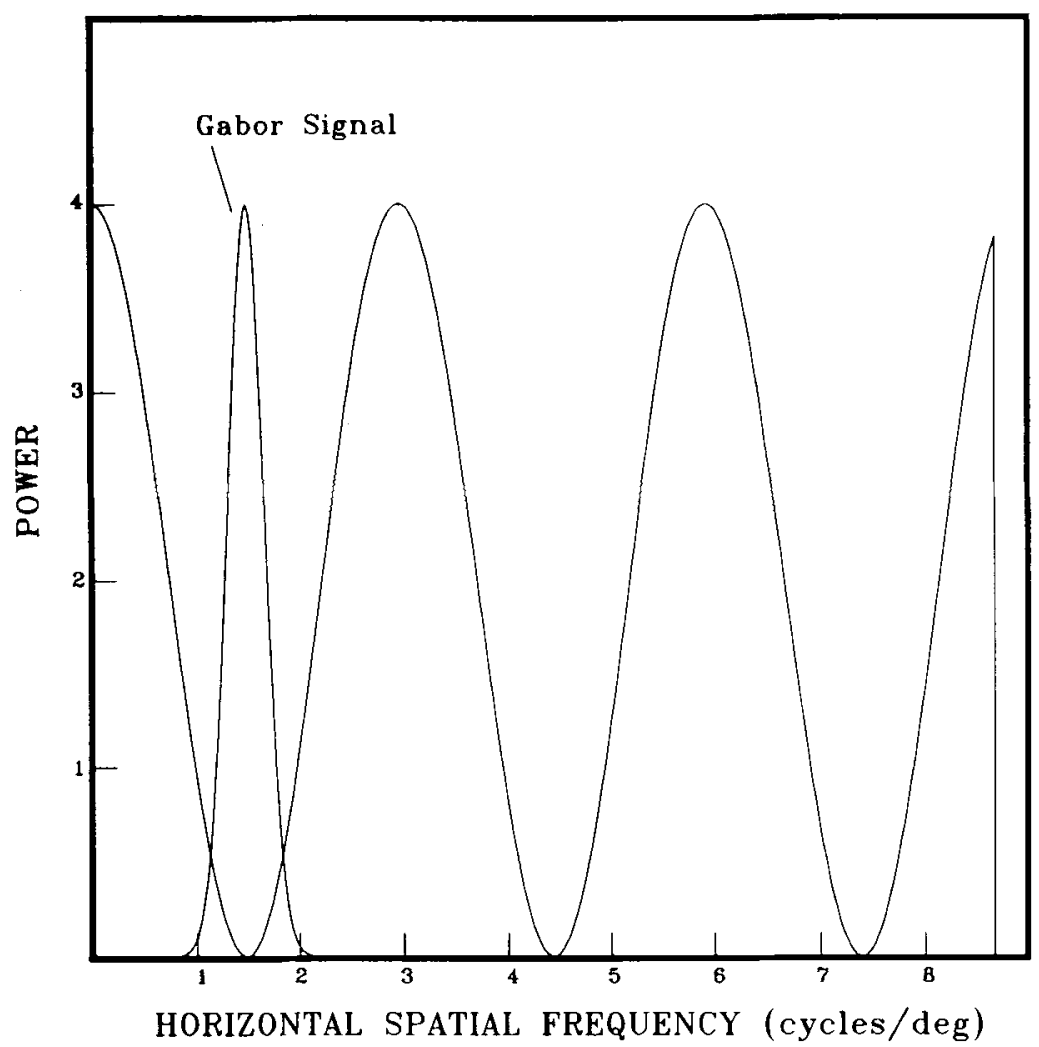

Figure 5. Variation in spectral power (arbitrary units) for the sum of left- and righteye Gaussian noise fields ( $d_{\alpha}=d_{\beta}=0$ ) as a function of the horizontal spatial frequency variable only. Also shown is the corresponding horizontal component of the power density function of the Gabor signal.

$$
\begin{aligned}
H(\epsilon, \eta)= & A_{h},-\epsilon_{u} \leq \epsilon \leq \epsilon_{u} \text { and }-\eta_{u} \leq \eta \leq \eta_{u} \\
& 0, \text { elsewhere, }
\end{aligned}
$$

where $\epsilon_{u}$ and $\eta_{u}$ represent the upper limits of horizontal and vertical spatial frequency resolution in the observer. Thus, if $h_{\mathrm{L}}(x, y)$ and $h_{\mathrm{R}}(x, y)$ are the internal noises, the internal representation of the luminance pattern for $g(x, y)\left[d_{\alpha}=d_{\beta}=d_{y}=0\right]$ after summation becomes

$$
g(x, y)+g\left(x+d_{x}, y\right)+h_{\mathrm{L}}(x, y)+h_{\mathrm{R}}(x, y),
$$

and its 2-D power spectral density function becomes

$$
2 H(\epsilon, \eta)+G(\epsilon, \eta)\left[2+2 \cos \left(2 \pi \epsilon d_{x}\right)\right] .
$$

Thus, the presence of internally generated noise results in imperfect signal unmasking in the $N_{d} S_{0}$ condition.

\section{The Phase Vector Model}

Consider a spatial frequency channel centered on the spatial frequency of the signal. If that channel is sufficiently narrow, the Gaussian noise passing through the channel can be considered as a sinusoidal signal whose frequency is equal to that of the channel but whose phase and amplitude are random. Such a signal can be represented as a vector in a 2-D space, with its length repre- senting the signal's amplitude and the angle it forms with the horizontal axis representing the signal's phase. Figure 6 (left side) illustrates such a vector for the left eye. Recall that the Gaussian noise presented to the right eye is shifted by a distance equal to half the wavelength of the Gabor signal and, hence, by half a cycle in the channel we are considering. Therefore, the vector representation of the output for the right eye will be a vector whose length is equal to that of the left eye's but whose phase is reversed by $180^{\circ}$. Now, when a signal in cosine phase is added to the left and right eyes (see Figure 6, right side), the resultant vectors are shifted in each eye so that they are no longer $180^{\circ}$ out of phase. Thus, binocular unmasking could result from a comparison of phase angles in the two eyes at the signal's frequency. In the $N_{d} S_{0}$ condition, an interocular phase difference not equal to $180^{\circ}$ would indicate the presence of a signal, whereas an interocular phase difference exactly equal to $180^{\circ}$ would indicate that no signal was embedded in the noise. In the $N_{d} S_{d}$ condition, however, the signal is also $180^{\circ}$ out of phase in the two eyes. Therefore, irrespective of whether or not the signal is added, the interocular phase difference remains at $180^{\circ}$. Thus, in the control condition, interocular phase difference could not discriminate between the presence and absence of a signal. 


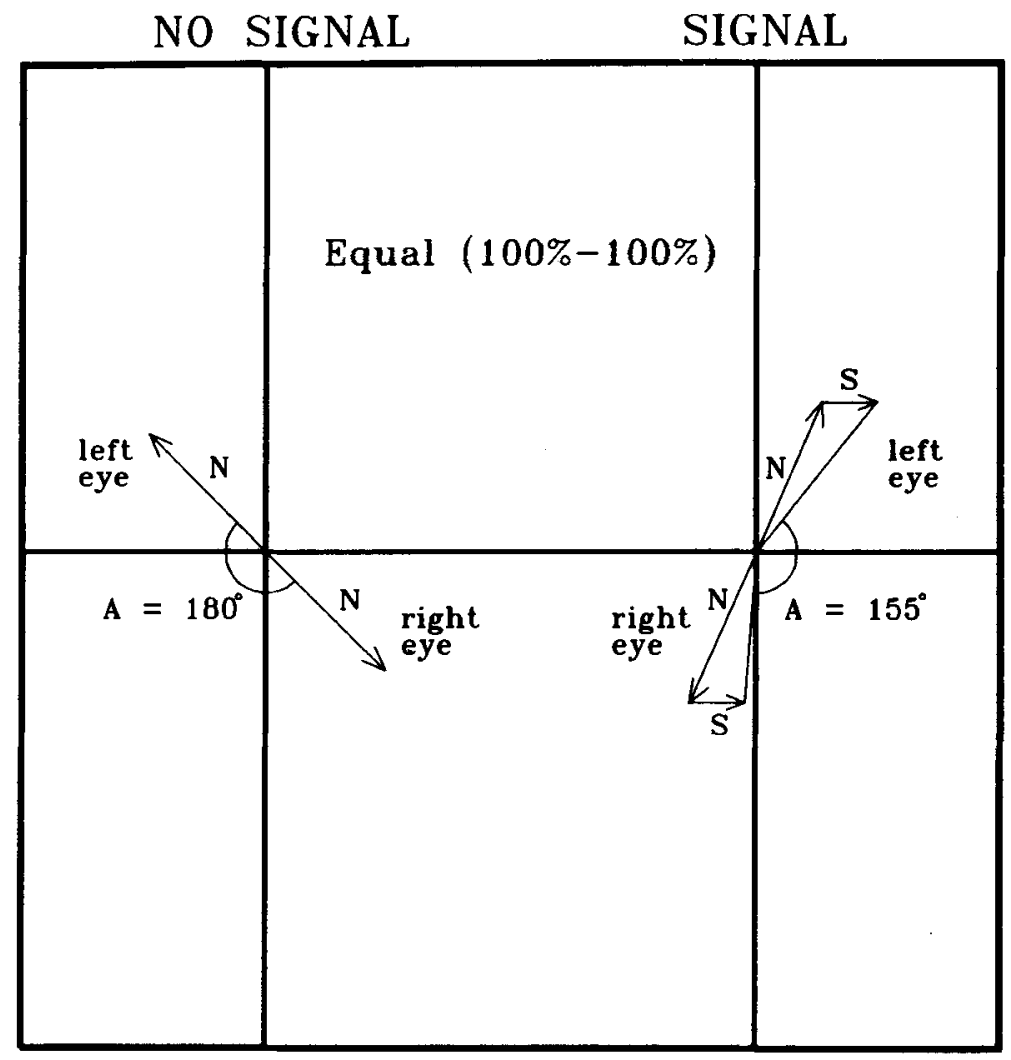

Figure 6. Vector diagram illustrating how interocular phase is shifted away from $180^{\circ}$ by the addition of a signal.

Again, without the presence of internally generated noise, the vector model would predict levels of signal detection in the $N_{d} S_{0}$ condition that are orders of magnitude better than those shown by observers. Therefore, it is reasonable to introduce within this model internally generated independent Gaussian noises in each of the eyes.

\section{Predictions for Unequal Interocular Contrast}

In the reference experiment, the RMS contrast of signal and masker in the right eye was $100 \%$ of that of the RMS contrast in the left eye. In two other experiments, the RMS contrast of both signal and masker in the right eye was set to $50 \%$ and $25 \%$ of the value in the left eye. In addition to these, two other experiments were conducted where the RMS contrast of signal and masker in both eyes was set to either $50 \%$ or $25 \%$ of the RMS contrast in the reference experiment.

Consider now what happens according to the summation model when contrast in the right eye is reduced by $50 \%$. If $r$ represents the ratio of the reduced to the original contrast ( $r=.5$, in this case), the summated pattern for the noise becomes

$$
g(x, y)+r g\left(x+d_{x}, y\right)+h_{\mathrm{L}}(x, y)+h_{\mathrm{R}}(x, y),
$$

and its 2-D power spectral density function becomes

$$
\left(1+r^{2}\right) A_{g}+2 A_{h}+2 r A_{g} \cos \left(2 \pi \epsilon d_{x}\right) .
$$

The luminance distribution for the summated Gabor signal in the experimental condition becomes

$$
(1+r) A_{j} \cos \left[2 \pi \epsilon_{s}\left(x-x_{0}\right)\right] \exp \left[-a^{2}\left(x-x_{0}\right)^{2}-a^{2}\left(y-y_{0}\right)^{2}\right]
$$

and therefore its power spectrum profile is reduced to $(1+r)^{2} / 4$ of its original value. Figure 7 (top panel) presents the power spectral density functions for both signal and noise along the horizontal spatial frequency axis for the summated inputs from the two eyes for the reference experiment. The middle panel presents the same functions for one unequal contrast experiment $(100 \%$ $50 \%$ ). Also shown are the same functions for the experiment in which the RMS contrast in both eyes was reduced by $50 \%$. In all cases, it was assumed that the amplitude of the internal noise was $10 \mathrm{~dB}$ lower than that of the external noise in the reference experiment. Figure 7 shows that the signal-to-noise ratio for the summated inputs is the largest for the reference experiment $(100 \%-100 \%)$, next largest for $50 \%-50 \%$, and smallest for $100 \%-50 \%$. Thus, the summation model predicts that BMLDs should be smallest in the unequal contrast case. We will now show that the vector model predicts a different rank order.

Figure 8 presents vector diagrams for the three cases discussed above. Note that in the $100 \%-50 \%$ case the lengths of both the right-eye noise vector and right-eye 


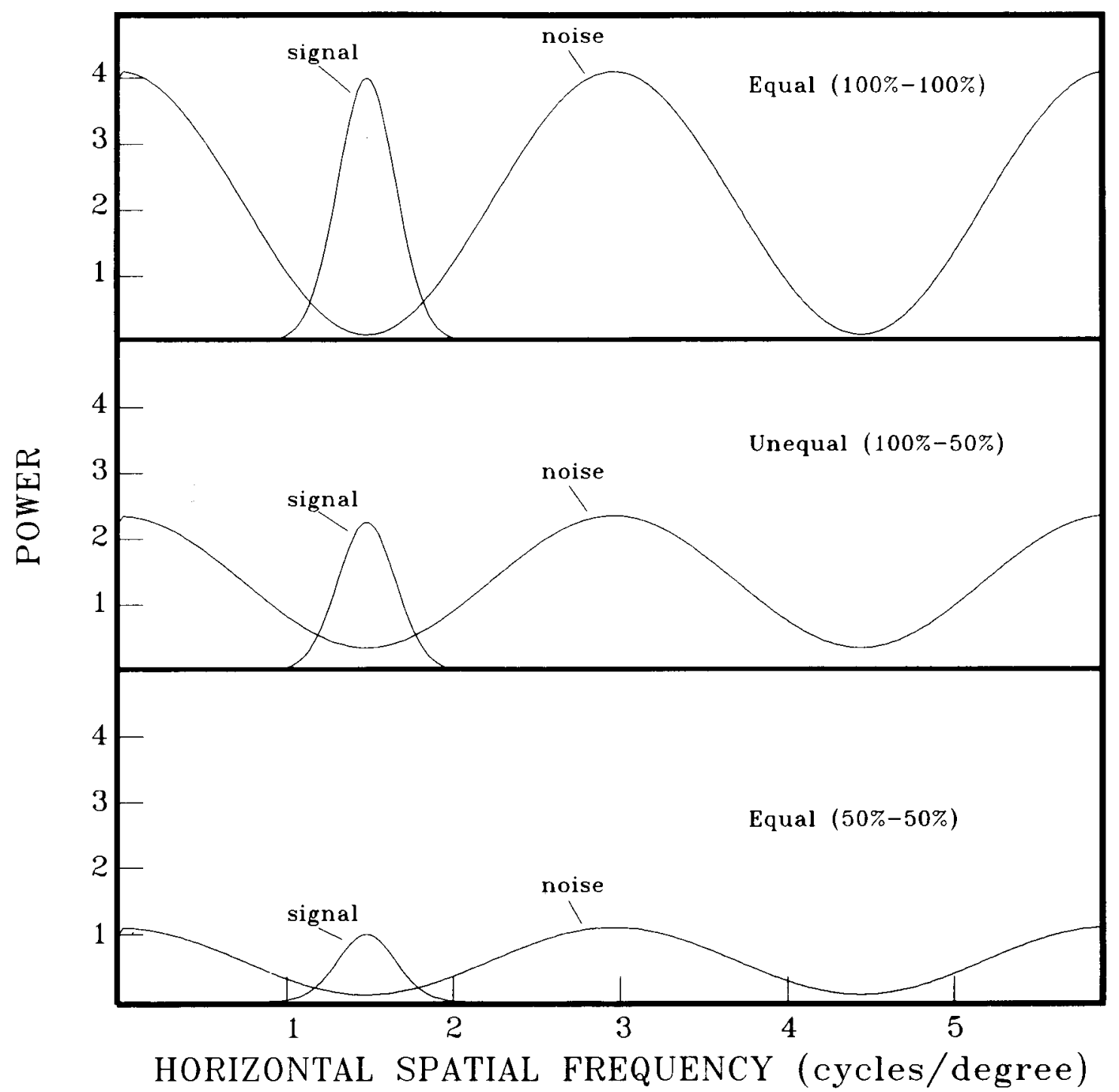

Figure 7. Power spectral density functions along the horizontal spatial frequency axis for the summed input of corresponding points from the two eyes for the reference experiment (upper panel), and for unequal-contrast $100 \%-50 \%$ (middle panel) and reduced-contrast 50\%-50\% (lower panel) experiments. In all cases, the masker in the right eye was shifted 20.3 minutes of arc to the right relative to the left eye masker creating the notch in the spectrum of the summed maskers; no shift was introduced for the Gabor signals. Independent internal noises with spectral levels $\mathbf{1 0} \mathrm{dB}$ lower than that of the external noise in the reference experiment are also assumed.

signal vector are exactly half those of the corresponding left-eye vectors. Clearly, reducing the size of the external noise and signal vectors by the same factor does not change the phase angle of the resultant vector, no matter what the size of the reduction is, providing that the vectors are not reduced to zero. Therefore, without internal noise, all three experiments should yield equivalent results.

The effect of internal noise on phase angle is illustrated by the circles surrounding the tips of the noise vectors in the no-signal interval and the signal + noise vector in the signal interval. The points along the perimeter of these circles illustrate possible locations for the tip of the vector produced by the addition of random internal noise of uniform vector length. Possible angular shifts introduced by this internal noise vector can be determined by drawing lines from the origin to the perimeter of the circle. The degree of angular change introduced by the addition of internal noise depends on the length of the vector to which it is added. Therefore, the variability in interocular phase angle produced by internal noise for both the noise and the signal + noise distributions increases as we proceed from equal $(100 \%-100 \%)$ to unequal $(100 \%-50 \%)$ to equal $(50 \%-50 \%)$. Figure 9 plots theoretical distribu- 
NO SIGNAL SIGNAL

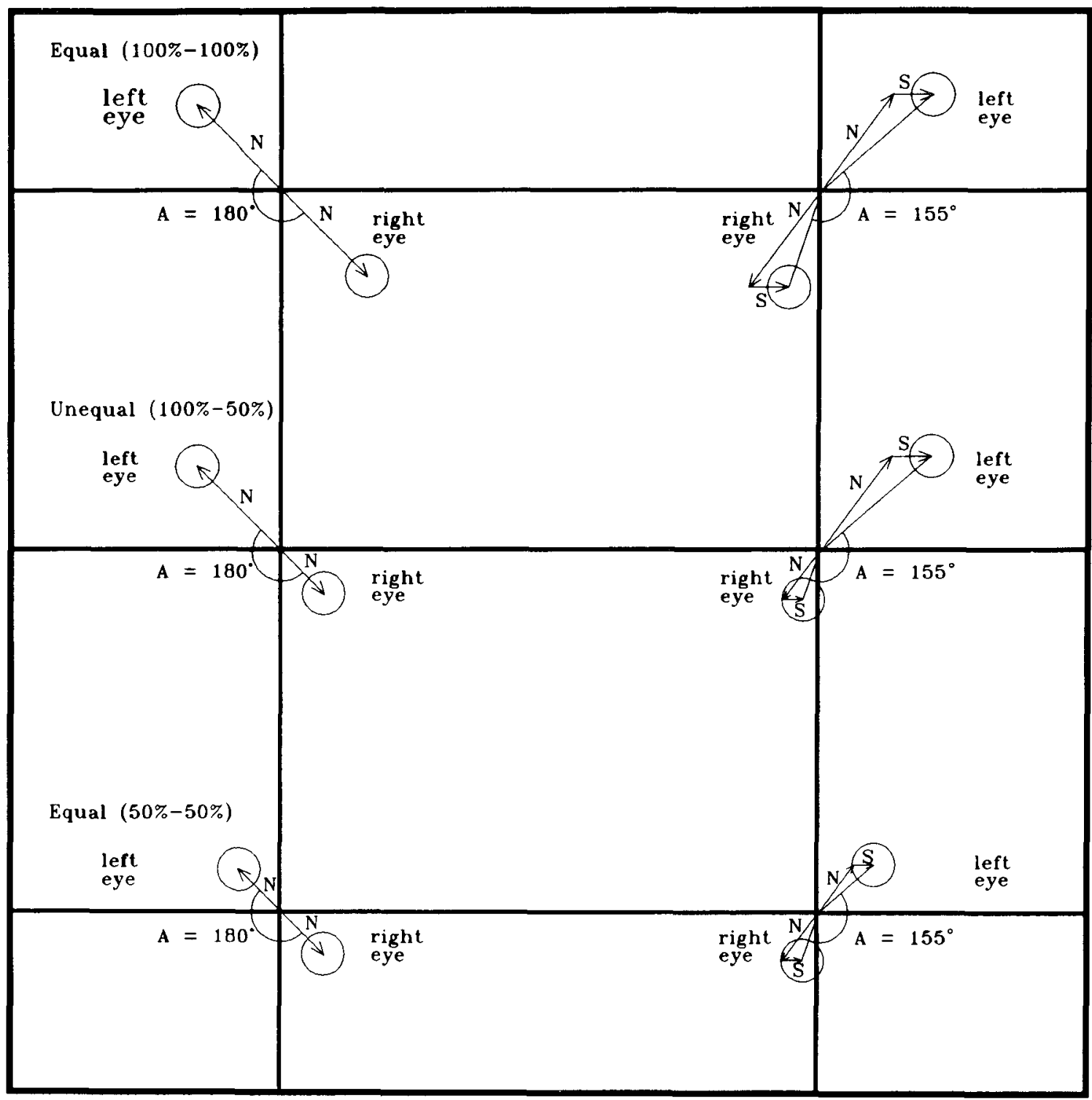

Figure 8. Vector diagrams illustrating how interocular phase is shifted away from $180^{\circ}$ by the addition of the signal for the three types of displays described in Figure 7. The points along the perimeter of the circles surrounding the tips of the vectors specify possible locations for the vectors' tips produced by the addition of random internal noise of uniform vector length.

tions of phase angles (see Appendix A) observed for these three cases. In these calculations, it was assumed that the amplitude of the internal noise was $10 \mathrm{~dB}$ less than that of the external noise in the reference experiment. Note that the variability in phase angle follows the expected order. Therefore, we would predict BMLDs of declining size as we go from $100 \%-100 \%$ to $100 \%-50 \%$ to $50 \%-50 \%$. Clearly, the two models predict different orderings of these three experimental conditions with respect to the size of the BMLD; these predictions were tested as described below.

\section{METHOD}

\section{Observers}

Two males (B.S., one of the authors, and D.B.) and 1 female (J.C.) served as observers in each of the experiments reported here. All had normal or corrected-to-normal acuity and normal stereo vision; all were experienced psychophysical observers. J.C. and D.B. were unaware of the hypothesis under investigation.

\section{Apparatus and Stimuli}

The displays, examples of which are shown in Figure 1 (middle and lower panels), were produced as 8-bit images by an IBM AT computer equipped with an ITI FG-100 image processor with 


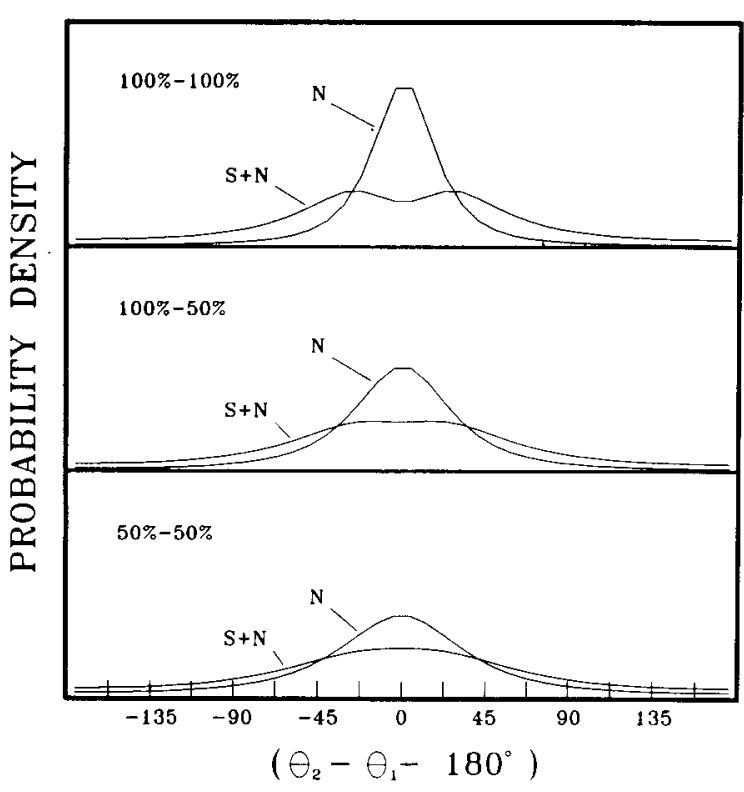

Figure 9. Theoretical distributions of the extent to which the difference between left- and right-eye vectors differs from $180^{\circ}$ for the reference experiment $(100 \%-100 \%)$ and two comparison experiments $(100 \%-50 \%$ and $50 \%-50 \%)$. In these calculations (see Appendix A), it was assumed that the spectrum level of the independent internal noises added to the left and right eyes was $10 \mathrm{~dB}$ below that of the noise in the reference experiment.

$512 \times 512$ pixel spatial resolution, and were presented through a rectangular aperture on a black-and-white 9-in. TV monitor (Panasonic WV 5370). The observers saw these displays through a simplelens stereoscope (Ogle, 1961, p. 99) equipped with neutral density filters, an individual pixel subtending 3.38' of visual angle. The left-eye display consisted of a $140 \times 140$ pixel field of 2-D Gaussian noise surrounded by a 15-pixel-wide frame of 2-D uniform noise (bandwidth $=8.7 \mathrm{cpd}$ in both cases); the right-eye image consisted of the horizontally right-shifted version $\left(d_{x}=-6\right.$ pixels or $\left.20.3^{\prime}\right)$ of the same Gaussian noise, also encased in a square noise frame identical to that presented to the left eye. Luminance, linearized via lookup tables, was in the range $0.2 \mathrm{~cd} / \mathrm{m}^{2}$ (gray level $=0$ ) to $9.6 \mathrm{~cd} / \mathrm{m}^{2}$ (gray level $=255$ ). The noise frame resulted from adding uniform random deviates from -40 to +40 to the mean pixel luminance $(\mu=128)$; its RMS contrast $\left(\mathrm{RMS}=\left[\Sigma\left(L_{i}-L_{\mathcal{u}}\right)^{\mathbf{2} / n}\right]^{1 / 2}\right)$, where $L_{u}$ is the mean luminance and $\mathrm{L}_{\mathrm{i}}$ is the individual pixel luminance) was $1.9 \mathrm{~cd} / \mathrm{m}^{2}$. The Gaussian fields were generated by rescaling a $140 \times 140$ matrix of random normal deviates with standard deviation equal to 80; the numbers in this table were then peakclipped at 1.6 standard deviations to keep them within the range -128 to +127 , and the final gray level values were obtained by adding 128 to each such number. The mean luminance of these Gaussian fields $(\mu=128)$ was $4.8 \mathrm{~cd} / \mathrm{m}^{2}$. In the reference experiment $(100 \%-100 \%)$, in which the left- and right-eye Gaussian noises were presented at full amplitude, their RMS contrast after peakclipping was $2.7 \mathrm{~cd} / \mathrm{m}^{2}$.

Because luminance resolution was limited to 8 bits, some distortion occurred for low-RMS gratings. For example, consider the grating whose wavelength was 12 pixels, and whose gray level value is given by $4 \cdot \cos (2 \pi i / 12)$, where $i$ is pixel location, with $i=0$ being the center of the waveform. The gray levels for 1 cycle of this waveform for pixel locations $0-11$ are $4,3,2,0,-2,-3$, $-4,-3,-2,0,2,3$. A truly continuous waveform would have an RMS value of 2.83 gray levels. Because gray levels are limited to the integer values above, the actual RMS value is 2.65 . More- over, a Fourier analysis of this waveform shows that there is some energy at the higher harmonics. However, the amount of energy at these higher harmonics is at least $20 \mathrm{~dB}$ lower than at the fundamental frequency. Hence, it is unlikely that the higher harmonics are contributing to detection. For higher amplitude gratings, the agreement between the theoretical RMS and the actual RMS is considerably better. For example, the RMS value for the continuous version of $128 \cdot \cos (2 \pi i / 12)$ is 90.51 gray levels, whereas the RMS value of its discrete version is 90.57 . Correspondingly, the relative sizes of the distortion products are smaller. It should also be noted that some distortion was introduced by peak-clipping. However, the magnitudes of these distortion products were small relative to both signal and noise. Therefore, they are unlikely to have affected performance. In sum, the major consequence of the digitization of gray levels appears to be that the theoretical RMS luminance of the signal may differ somewhat from its actual RMS luminance for low-amplitude signals. In our calculations, we used the theoretical RMS values.

As in our previous studies, the location at which a Gabor signal (see Figure 1, top panel) was added to the right side of the display defined two conditions. In the experimental condition $\left(N_{d} S_{0}\right)$, the right signal was added to the noise at the location exactly corresponding to that of the left signal-in both cases, the signal centers coincided with the displays' (Figure 1, lower panel). In the control condition $\left(N_{d} S_{d}\right)$, the right signal was added to the noise following a horizontal shift equal in extent and direction to that of the noise. In both conditions, the signals were added to the noise in one of the two display pairs making up each trial. The Gabor signal (Figure 1, upper panel) had a 2-D half-width Gaussian space constant of 18 pixels (to $1 / e$ ); the phase of the grating was $0^{\circ}$, its orientation vertical, and its wavelength 12 pixels, which corresponded to a spatial frequency of $1.48 \mathrm{cpd}$. The gray levels of each pixel in the Gabor signal in the noise were given by $A_{j} \cos \left[2 \pi\left(x-x_{0}\right) /\right.$ $\lambda] \exp \left\{-a^{2}\left[\left(x-x_{0}\right)^{2}+\left(y-y_{0}\right)^{2}\right]\right\}$, where $a$ is the reciprocal of the space constant, $\lambda$ the signal wavelength, $A_{j}$ its gray level amplitude, and $x_{0}, y_{0}$ the field center. For both conditions, four levels of signal RMS were used (the latter was computed over the pixels located within the 1/e boundary of the signal's Gaussian envelope). Pilot sessions of about 30-min duration were conducted to select the signal levels appropriate for each observer. In addition to the reference experiment, four other experiments were conducted in which the RMS contrast of both signal and noise was changed. In the experiment labeled $50 \%-50 \%$, the RMS contrast of signals and Gaussian noises in both fields was reduced to $50 \%$ of its value in the reference experiment. In the experiment labeled $25 \%-25 \%$, the RMS contrast of signals and noises in both fields was reduced to $25 \%$ of its full value. In the experiment labeled $100 \%-50 \%$, the left-eye Gaussian noise and signal were presented at full amplitude, while the RMS contrast of the right-eye Gaussian noise and signal was reduced by $50 \%$. Finally, in the experiment labeled $100 \%-25 \%$, the contrast of the Gaussian noise and signal in the right eye was only one fourth that of their counterparts in the left eye. Thus, in all cases, the signal-to-noise ratio in the right eye was identical to that in the left eye.

\section{Procedure}

In all five experiments, the observers sat in a darkened room, looking through the stereoscope at the apertured portion of the TV screen. Initially, the fused display consisted of the noise frame encasing a blank area at whose center was a dark fixation spot, the blank region being identical in extent and mean luminance to the Gaussian noise fields to be presented during the trial. The square frame was employed to aid fusion prior to stimulus presentation. Thus, prior to and in between the two stimulus intervals, the observer saw a square frame with a fixation spot in the center. A buttonpress triggered the presentation of a 1-sec test display presented within the frame. Because the right-eye test display was dis- 
placed to the right, the observers saw the Gaussian noise field as appearing behind the frame. After the 1-sec presentation, the fixation display was again presented for $0.5 \mathrm{sec}$ and followed by another 1-sec test display. Each of the two intervals contained different pairs of Gaussian noises (randomly selected from a set of 104 such pairs) presented within the frame, the signals being always added to one of the two noise pairs. All observers reported seeing two samples of stationary visual noise appear behind the square frame during the two 1 -sec intervals. When the target was presented at high contrast levels in one of the intervals in condition $N_{d} S_{0}$, it appeared to "float" in front of the background on the same plane as that of the square frame. When the target was presented at high contrast levels in $N_{d} S_{d}$, it appeared to be embedded in the noise background. None of the subjects reported seeing any motion, nor did they experience diplopia in any of the conditions. The observer's task was to indicate, by pressing the appropriate key on a response box, in which of the two test displays the signal had been added to the Gaussian noise fields. A computer-generated tone followed this keypress when the correct interval had been chosen. A block of trials consisted of displays of the same condition, the signals' contrast taking any of four RMS values in random order, subject to the constraint that each such value was employed an equal number of times. For each experiment, each observer served in 832 trials, 104 trials being collected per level of signal RMS at each condition. These trials were delivered in eight blocks; order of block presentation as a function of condition was randomized independently for each observer. The reference experiment was run first, and the order in which the remaining experiments were administered was randomized independently for each participant, who all completed 4,160 trials. It should be noted that we have repeatedly observed that the effects of practice in this type of experiment are marginal at best.

\section{RESULTS}

Psychometric functions were obtained from each of 3 observers for the $N_{d} S_{0}$ and $N_{d} S_{d}$ conditions of each experiment. A reminder: In the reference experiment $(100 \%-100 \%)$, the RMS contrasts of both the Gaussian noise and the Gabor signal were identical in both eyes. In two other experiments, the RMS contrasts of both noise and signal were identical, but were either $50 \%(50 \%-50 \%)$ or $25 \%(25 \%-25 \%)$ of the reference experiment's RMS contrast. In a fourth experiment, the RMS contrast of the left eye remained at reference levels, whereas the RMS contrasts of both signal and noise in the right eye were set at $50 \%$ of the left eye value $(100 \%-50 \%)$. In the fifth experiment, the right eye contrasts for both signal and noise were set at $25 \%$ of the left eye value $(100 \%-25 \%)$.

Percent correct detection responses as a function of signal-to-noise ratio in decibels are shown in Figure 10 for the 3 observers when both left- and right-eye fields were at full contrast $(100 \%-100 \%)$. Clearly, for abovethreshold RMS values the Gabor signals were more easily detected in the $N_{d} S_{0}$ (filled symbols) than in the $N_{d} S_{d}$ condition. By defining threshold as signal-to-noise ratio corresponding to $75 \%$ correct, and the BMLD as the performance difference (measured in decibels) between the two conditions at threshold, the estimated BMLDs were 14.5, 14.4, and 4.1 dB for Observers B.S., J.C., and D.B., respectively. Figure 11 shows the performance of these observers as a function of signal-to-noise ratio in

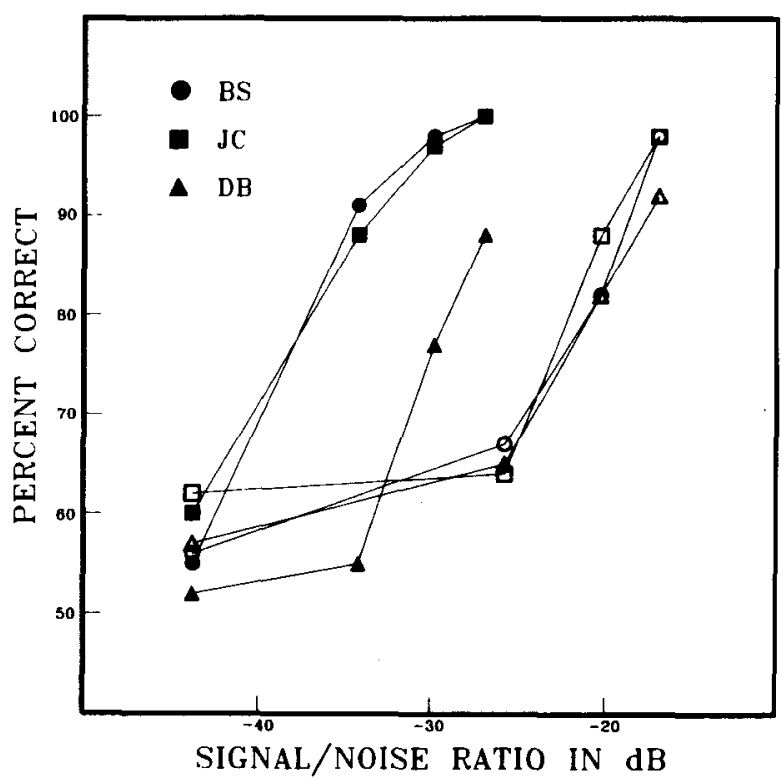

Figure 10. Percentage of correct responses as a function of signalto-noise ratio in decibels for 3 observers in the reference experiment. Filled symbols represent the $N_{d} S_{0}$ condition; open symbols stand for the $N_{d} S_{d}$ condition.

the $N_{d} S_{d}$ condition of all five experiments. There is no indication that performance depended on the level of interocular contrast. The extent of binocular unmasking was therefore evaluated with reference to the average threshold across the $N_{d} S_{d}$ baseline condition for each subject.

Figure 12 shows how the BMLD varied with RMS contrast for the experiments in which the interocular contrast was the same (unfilled circles) and different (filled circles). While large individual differences can be noted, as we also found in previous experiments, it can be generally said that performance was better with the equalcontrast displays than with the unequal-contrast ones. The smooth curves are the predictions of a two-parameter linear summation model in which one of the parameters is the spectrum level of the internal noise relative to that of the full-scale external noise, and the other parameter is the bandwidth of a 2-D Gabor filter centered at the signal's peak frequency (see Appendix B). The fits for the equal-contrast conditions are quite good for all 3 subjects and for the average data. The fits for the unequal-contrast experiments are good for Subjects B.S. and D.B. and about $2 \mathrm{~dB}$ too high for Subject J.C. The predicted functions for Observer B.S. assume a Gaussian filter with a bandwidth (to the $1 / 2$ power points) of $0.23 \mathrm{cpd}(0.23 \mathrm{oc}-$ taves), with the spectrum level of the internal noise being $13 \mathrm{~dB}$ below that of the full-scale external noise. The fit for Subject J.C. required a bandwidth of $0.44 \mathrm{cpd}(0.43$ octaves), with the spectrum level of the internal noise being $23 \mathrm{~dB}$ below that of the full-scale external noise. The bandwidth for Subject D.B. was 1.75 cpd (1.96 octaves), with the spectrum level of the internal noise $20 \mathrm{~dB}$ below 


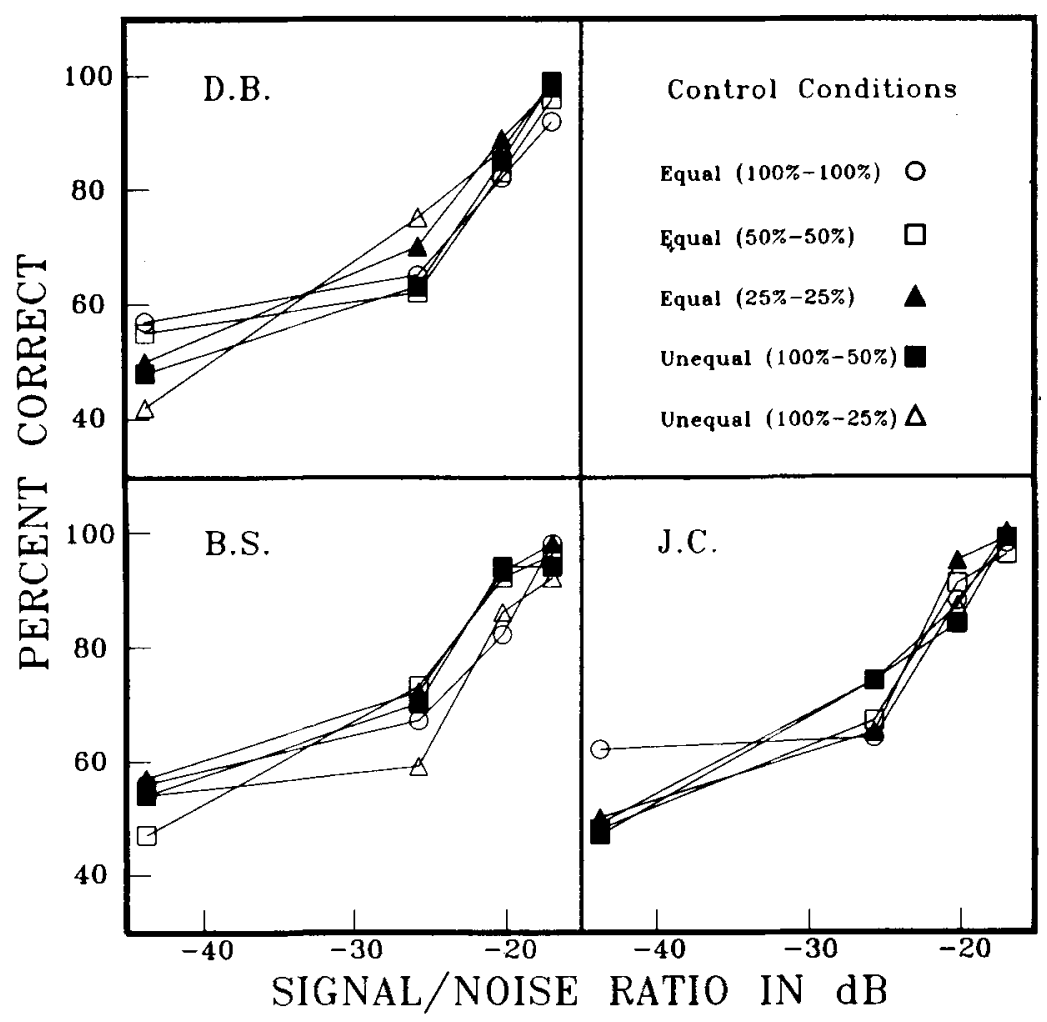

Figure 11. Percentage of correct responses as a function of signal-to-noise ratio for the 3 observers in each of the five $N_{d} S_{d}$ control conditions.

that of the external noise. The bandwidth for the average data was 0.64 cpd $(0.63$ octaves), with the internal noise set $16.5 \mathrm{~dB}$ below that of the full-scale external noise.

No attempt was made to fit the vector model to the data, because the vector model predicts that the $50 \%-50 \%$ experiment should induce a smaller BMLD than that of the $100 \%-50 \%$ experiment, and because the $25 \%-25 \%$ experiment should produce a smaller BMLD than is obtainable in the $100 \%-25 \%$ experiment. The results for all 3 subjects were in the opposite direction. If the vector model were correct, the only way this could happen is if the internal noise was so intense that it effectively overwhelmed the phase shift caused by the presence of the signal. If this were to occur, then the probability, say, that the $100 \%-50 \%$ experiment should have a smaller BMLD than the 50\%-50\% experiment would be approximately 0.5 . The same should hold true for the $100 \%-25 \%$ versus $25 \%-25 \%$ comparison. Therefore, if the vector model were correct, but with a very large amount of internal noise, then $p(100 \%-50 \%>50 \%-50 \%)=1 / 2$, and $p(100 \%-25 \%>25 \%-25 \%)=1 / 2$. The probability that all 3 subjects should show the pattern observed in Figure 12 then is $1 / 64$. Note also that this is a conservative estimate of the true probability. If the true probability of $100 \%-50 \%>50 \%-50 \%$ were actually close to $1 / 2$, the internal noise would be so large that the BMLDs in both conditions would be close to zero. Figure 12, however,

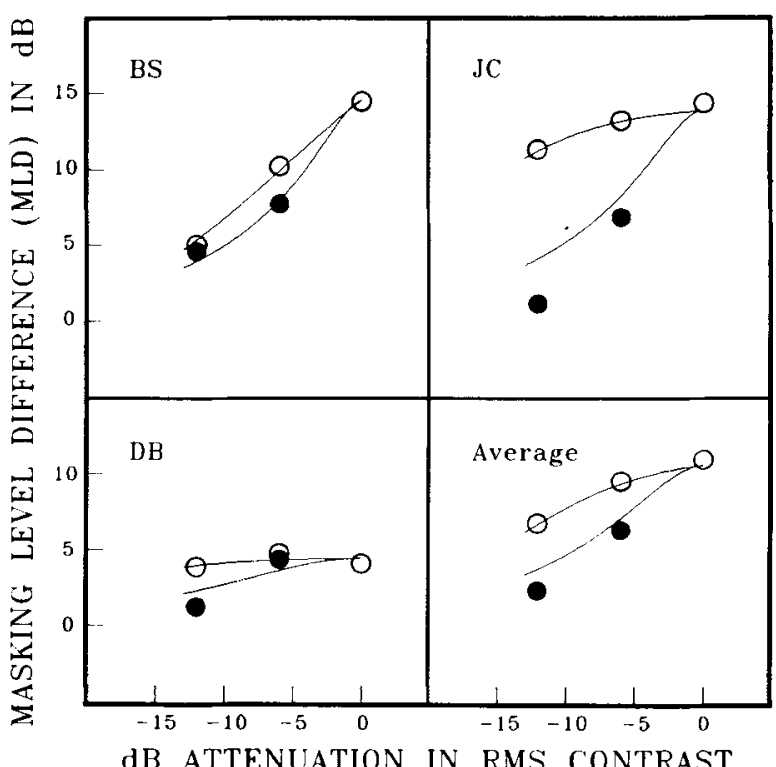

Figure 12. Binocular masking level differences (BMLD) for 3 observers (average BMLDs are also shown) as a function of the attenuation in the RMS contrast of the experimental displays relative to the values of the reference experiment. Open circles represent the three equal conditions; closed circles represent the two unequal conditions. The smooth curves are the prediction of the summation model. 
indicates that BMLDs for the equal conditions were quite a bit larger than zero. Therefore, the probability of obtaining the observed pattern of results from the 3 subjects, assuming that the vector model is correct, is considerably less than $1 / 64$. The results, therefore, clearly reject the vector model.

\section{DISCUSSION}

Two different types of models have been proposed over the years to explain unmasking effects in both audition and vision. The first class of models assumes that perceptual systems first process the information coming from the two eyes or the two ears separately. Within each eye or each ear, summary statistics of dimensions of the stimulus pattern are computed. For example, each eye could analyze the pattern in terms of its orientation and spatial frequency composition. In such a model, at any given orientation and spatial frequency, the right eye might compute the amplitude and phase of that spatial frequency component. A similar decomposition and analysis would occur in the other eye. Binocular processing might then consist of interocular comparisons of the amplitude and phase values of these components. If noise and signal + noise trials produced either interocular amplitude differences or interocular phase differences, these differences could then be used to discriminate between signal and nosignal trials. The phase-vector model is an example of such a theory, and it predicts that performance in the unequal-contrast conditions should be better than in the equal-contrast conditions.

The second class of models assumes that, for the purposes of unmasking, the visual or auditory patterns in the two eyes or ears are combined before further processing. In other words, a Cyclopean eye is formed to obtain a single representation of the visual world. The summation model that we propose falls into this class because it assumes that the patterns in the two eyes are added together to form a single visual representation, with summary statistics, such as those described above, computed on the Cyclopean pattern. If noise and signal + noise trials differ with respect to specific summary statistics computed on the summated pattern, this difference could be used as a basis for distinguishing between the two alternatives.

More specifically, in the present model it is assumed that a multitude of Cyclopean eyes can be formed by adding together the luminance patterns from the two eyes after they have been shifted relative to each other either horizontally or vertically, or both. These shifts are accomplished by (1) vergence movements or (2) they are internally generated $\left(d_{\alpha}, d_{\beta}\right)$. In general then, if $f_{\mathrm{L}}(x, y)$ is the luminance pattern presented to left eye, and $f_{\mathbf{R}}(x, y)$ is the pattern presented to the right eye, a Cyclopean eye is defined by

$$
f_{\mathrm{L}}(x, y)+f_{\mathrm{R}}\left(x+d_{\alpha}+d_{x v}, y+d_{\beta}+d_{y v}\right),
$$

where $d_{\alpha}$ is the internal horizontal displacement, $d_{x v}$ represents the degree of horizontal displacement due to vergence movements, $d_{\beta}$ and $d_{y v}$ are their vertical counterparts, and the point $(x=0, y=0)$ specifies the geometric center of each luminance pattern. Note that under normal viewing conditions, $f_{\mathrm{L}}$ and $f_{\mathrm{R}}$ would be highly correlated patterns, and it is assumed that the extent of possible internal shifts is limited (e.g., to Panum's area). In the present model, it is assumed that a spectral analysis is conducted on one or more of the possible Cyclopean eyes. If the spectral patterns differ for noise and signal + noise distributions in any such eye, this difference will be used to discriminate between these two alternatives.

It is also important to note that it does not matter whether vergence movements image the background or the signal on corresponding points. In the present experiments, given the fixation conditions, it is assumed that the signal would fall on corresponding points and that the background noise would be displaced on the retinas. Therefore, the Cyclopean eye defined by $\left(d_{\alpha}=d_{s}=0\right)$ would produce a notched noise spectrum and a summated signal. However, if vergence movements occurred, such that the background now fell on corresponding points $\left(d_{x v}=-d_{x}\right)$ so that $N_{d} S_{0}$ and $N_{d} S_{d}$ effectively become $N_{0} S_{d}$ and $N_{0} S_{0}$, the Cyclopean eye defined by $\left(d_{\alpha}=d_{x}\right.$, $d_{\beta}=0$ ) would still have the notched noise spectrum and summated signal.

With respect to the present experiments, the summation model predicts that, unless the internal noise is very high (see Appendix B), performance should be poorer in the unequal-contrast case than in the equal-contrast case. The experimental data supported this prediction, rather than the prediction of the vector model. Furthermore, the quantitative predictions of a two-parameter summation model provide a good fit for all of the subjects in the equalcontrast experiments and a good fit for 2 of the subjects in the unequal-contrast experiments. For the 3 rd subject, J.C., the predicted values in the unequal-contrast case were slightly higher $(1.5 \mathrm{~dB}$ at $100 \%-50 \%$ and $3 \mathrm{~dB}$ at $100 \%-25 \%$ ) than the obtained values. Thus, the summation model not only can account for the qualitative trends in the data but also provides a fairly good quantitative account.

The two parameters of the summation model were the bandwidth of the internal filter and the level of internal noise. Estimates of internal bandwidth ranged from 0.23 to 1.96 octaves, with the larger bandwidth associated with the oldest subject (D.B., age $=54$ years). Pichora-Fuller and Schneider (1991) have shown that estimates of bandwidth for elderly subjects in an auditory equivalent of the present model are larger than those of young subjects. It is conceivable that the larger bandwidth exhibited by D.B. represents a visual counterpart of this auditory aging effect; to explore this possibility, a systematic study concerning age-related differences in the occurrence of binocular unmasking is currently under way in our laboratories. It is also worth noting that our average bandwidth estimate is in the range of those found for human observers 
of low-contrast gratings (e.g., Sachs, Nachmias, \& Robson, 1971, 0.4 octaves; Legge \& Foley, 1980, 0.5 octaves; Watson, 1982, 0.5 octaves). Further parametric work will be required, however, to determine just how well the two-parameter model can account for binocular unmasking or whether some other model of the Cyclopean eye would provide a better description.

The test of the summation model attempted here lends, we believe, appreciable support to our account of binocular unmasking and is fully consistent with our previous investigations of this process. These investigations established that detections thresholds appeared to depend on the signal-to-noise ratio in the summated pattern. We showed in a variety of ways that whenever appropriate amounts of horizontal and vertical shifts were used to produce notches in the summated noise at particular spatial frequencies and peaks in the summated signals at the same spatial frequency, BMLDs were observed. Conversely, no evidence of binocular unmasking was ever found when the signals were "located" outside of the notches in the 2-D power spectrum of the summated noise.

It is important to note that, in the absence of visual noise, our paradigm reduces to a binocular summation paradigm, and our model becomes functionally equivalent to the binocular summation model proposed by Campbell and Green (1965). As such, it clearly predicts that binocular thresholds, with equal contrast in each eye, should be $\sqrt{2}$ lower than the corresponding monocular thresholds, a finding that is well documented in the literature (for reviews, see Blake \& Fox, 1973; Blake, Sloane, \& Fox, 1981). In a recent paper, Anderson and Movshon (1989) evaluated binocular summation for sinusoidal gratings that varied in the ratio of contrasts presented to the two eyes. Their study, then, is equivalent to our study without visual noise. They argued that their results were incompatible with the Campbell and Green model; they might therefore be seen as incompatible, by implication, with our model of binocular summation. However, they note that in order for the Campbell and Green model to work, it must be assumed that "the observer can identify and discount the internal noise arising from one eye when the signal is presented to the other" (p. 1115). Another way of rephrasing this condition is to say that the Campbell and Green model requires that the observer have independent access to each of the monocular channels, as well as to the binocular channel. In fact, Wolfe (1986) has argued for the existence of such separate and independent channels. According to such a notion, detection would occur whenever activity in any one of these channels reached threshold. Thus, if the grating was presented only to the right eye, detection would be based on the right-eye channel and not on the binocular channel, because the signal-to-noise ratio would be higher in the monocular channel than in the binocular channel. When the contrast in the two eyes is equal, however, detection will be based on the binocular channel because, under these conditions, the signal-to-noise ratio in the binocu- lar channel is $\sqrt{2}$ higher than in the monocular channel. For conditions of unequal contrast, detection would be based on the channel with the higher signal-to-noise ratio.

Figure 13 is a replotting of the data of Observer E. A. from Anderson and Movshon (1989) along with the predictions from our summation model. (Similar results would be obtained for the other observers.) The ordinate and abscissa of this figure represent the threshold contrasts for the right- and left-eye gratings, with the points lying on the ordinate and abscissa representing monocular viewing conditions. The ratio of the $y$-coordinate to that of the $x$-coordinate specifies the contrast ratio used in determining threshold. The solid line represents the predictions of the summation model (see Appendix B). When the contrast in one eye is much greater than the contrast in the other eye, the model predicts that the threshold for these unequal-contrast conditions should be equal to the monocular threshold. When, however, the signal-to-noise ratio in the binocular channel becomes larger than those in the monocular channels, then the contrast needed for threshold is less than that required for monocular detection. Note that the binocular summation model, with independent access to monocular and binocular channels, can provide a good account for the data. Furthermore, if one makes allowance for the occurrence of probability summation across channels, the abrupt transition from a monocular to the binocular channel would be smoothed considerably. The reason for this is that as the signal-tonoise ratio approaches equality in the monocular and binocular channels, detection could be based on the momentary value in either channel, leading to a degree of probability summation and a rounding of the abrupt transition in the predicted functions.

Legge (1984) has proposed a model of binocular interaction in which the signal from each eye is squared and integrated before summation occurs (see his Figure 4). This model clearly cannot account for the binocular unmasking data, because if the monocular signals are squared and integrated before summation there cannot be any cancellation and therefore any binocular unmasking. In general, any models that involve squaring or rectification before summation are incompatible with the results from binocular unmasking. Because Cogan's (1987) model involves rectification in the monocular channels, and because the fused binocular channel responds only when the monocular responses are in the same spatiotemporal phase interocularly, it also cannot handle the results from binocular unmasking experiments.

Although Anderson and Movshon (1989) propose a model for binocular summation that provides a good account of the classical data on binocular summation, their model is also unable to predict the results of the present unmasking experiments with unequal interocular contrast. They hypothesize that the visual system has a number of binocular channels that differ from one another with respect to the relative number of inputs from each eyethat is, they differ in the weight given to each eye's in- 


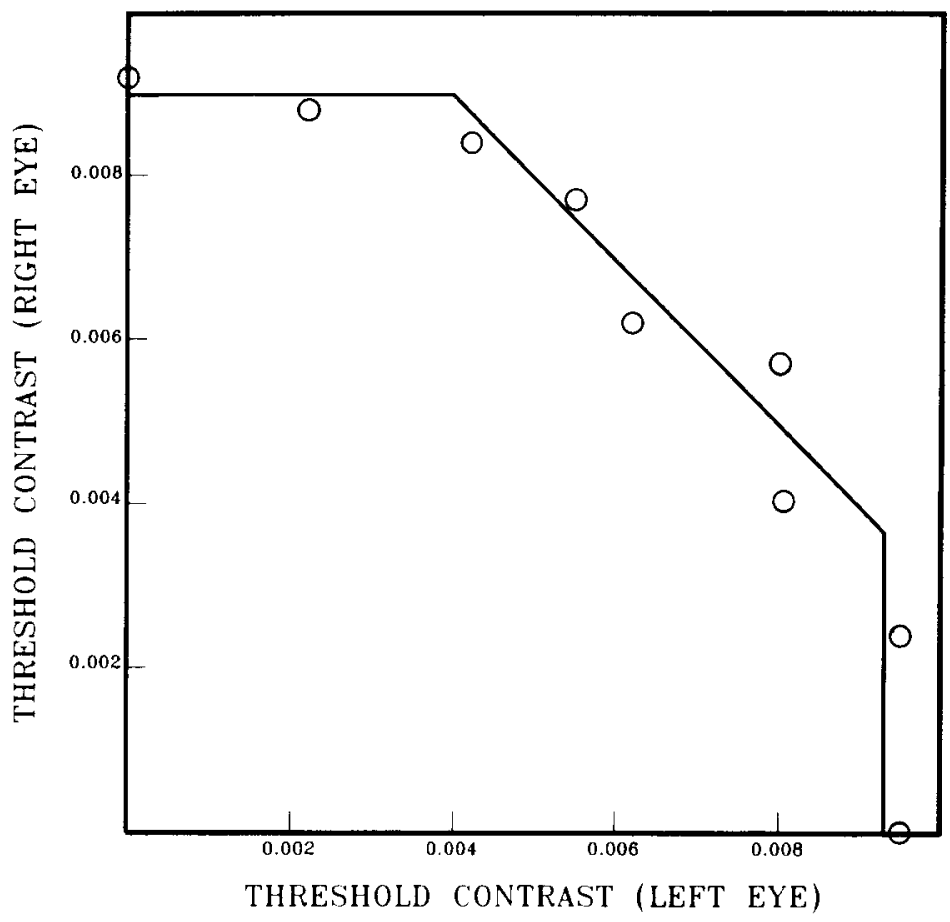

Figure 13. Binocular summation data for Observer E.A. from Anderson and Movshon (1989). The abscissa gives the contrast at threshold for the left-eye grating; the ordinate gives the contrast at threshold for the right-eye grating in a binocular situation. For instance, the point $(x=.0094, y=0)$ represents a monocular stimulus to the left eye; the point $(x=.0062, y=.0062)$ represents equal contrast in both eyes, and the point $(.008, .004)$ represents a contrast ratio between the two eyes of 2:1. Left and right gratings (spatial frequency $=2.6 \mathrm{cpd}$ ) were in phase. The solid line represents the predictions of our model, assuming (1) that the observer has independent access to the two monocular channels and the binocular channel and (2) that there are independent sources of internal noise in each monocular channel. The data points plotted here were taken from Figure $1 \mathrm{~A}$ in Anderson and Movshon (1989). (Reprinted with permission from Vision Research, Vol. 29, P. A. Anderson and J. A. Movshon, "Binocular combination of contrast signals," 1989, Pergamon Press.)

put. Thus, in their model there would be a binocular channel in which the left-eye signal would be weighted four times as much as the right-eye signal. Conversely, there should be another channel in which the weighting function is reversed. They, like us, assume linear summation within these channels, with detection occurring whenever activity in any one of these channels exceeds threshold. This means that when contrast of the left-eye signal is one fourth that of the right-eye signal, then in the channel where the left eye input is weighted four times more than the right-eye signal, both eyes will contribute equally after weighting has occurred. Thus, with shifted noise, cancellation will be virtually complete at some frequencies, and good binocular unmasking should occur in this channel. Another way of viewing their model is that the differential weighting function in this channel serves to equalize the two inputs. In this respect, it is like Durlach's (1972) equalization and cancellation model of binaural unmasking, in the sense that the amplitude (contrast) in each ear (eye) is equalized before subtraction or cancellation. Such a model, then, would predict that when the magni- tude of the external masker is much greater than that of the internal noise, the amount of unmasking should be the same for the $100 \%-100 \%$ condition as it is for the $100 \%-25 \%$ condition. Although this prediction of an equalization and cancellation model holds in the auditory realm (McFadden, 1968), the present data show clearly that it fails in vision. Therefore, we can conclude that a model that involves unequal weights applied to the monocular inputs before summation cannot account for the data.

Concerning the specification of the possible physiological embodiment of the summation process, electrophysiological studies of the striate cortex suggest that many binocular interactions can be accounted for in terms of linear summation of neural signals from the eyes (e.g., Ohzawa \& Freeman, 1986a, 1986b). Psychophysical evidence for the linearity of spatial-frequency-andorientation-selective binocular neurones in the human visual system is also available (e.g., Blake \& Levinson, 1977). Moreover, comparable bandwidths estimates are also reported in several electrophysiological studies of 
binocular cortical units (e.g., Albrecht, 1978; R. L. De Valois, Albrecht, \& Torrel, 1982; see also R. L. De Valois \& K. K. De Valois, 1988). Mechanisms such as these, characterized by the properties of binocularity, linearity, and relatively narrow tuning in the 2-D Fourier domain can thus be tentatively regarded as potential candidates for the mediation of the effects observed in our study.

\section{REFERENCES}

Abramowitz, M., \& STEgun, I. A. (1970). Handbook of mathematical functions. New York: Dover Press.

Albrecht, D. G. (1978). Analysis of visual form. Unpublished doctoral dissertation, University of California, Berkeley.

ANDerson, P. A., Movshon, J. A. (1989). Binocular combination of contrast signals. Vision Research, 29, 1115-1132.

BLAKE, R., \& Fox, R. (1973). The psychophysical inquiry into binocular summation. Perception \& Psychophysics, 14, 161-185.

BLAKE, R., LEVINSON, E. (1977). Spatial properties of binocular neurones in the human visual system. Experimental Brain Research, 27, 221-232.

Blake, R., Sloane, M., \& Fox, R. (1981). Further developments in binocular summation. Perception \& Psychophysics, 30, 266-276.

CAMPBell, F. W, \& Green, D. G. (1965). Monocular versus binocular visual acuity. Nature, 208, 191-192.

COGAN, A. I. (1987). Human binocular interaction: Towards a neural model. Vision Research, 27, 2125-2139.

Colburn, H. S., \&urlach, N. I. (1978). Models of binaural interaction. In E. C. Carterette \& M. P. Friedman (Eds.), Handbook of perception: Vol. 4. Hearing (pp. 467-518). New York: Academic Press.

Daugman, J. G. (1980). Two-dimensional spectral analysis of cortical receptive field profiles. Vision Research, 20, 847-856.

De Valois, R. L., Albrecht, D. G., \& Torell, L. G. (1982). Spatial frequency selectivity in macaque visual cortex. Vision Research, 22, 545-559.

DE VAloIS, R. L., \& DE VAloIs, K. K. (1988). Spatial vision. New York: Oxford University Press.

DuRLACH, N. I, (1972). Binaural signal detection: Equalization and cancellation theory. In J. Tobias (Ed.), Foundations of modern auditory theory (Vol. 2., pp. 369-462). New York: Academic Press.

Durlach, N. I., \& Colburn, H. S. (1978). Binaural phenomena. In E. C. Carterette \& M. P. Friedman (Eds.), Handbook of perception: Vol. 4. Hearing (pp. 365-466). New York: Academic Press.
Henning, G. B., \& Hertz, G. B. (1973). Binocular masking level differences in sinusoidal grating detection. Vision Research, 13, 2455-2463

Henning, G. B., Hertz, G. B. (1977). The influence of bandwidth and temporal properties of spatial noise on binocular masking-level differences. Vision Research, 17, 399-402.

JefFRESS, L. A. (1972). Binaural signal detection: Vector theory. In J. V. Tobias (Ed.), Foundations of modern auditory theory (pp. 349368). New York: Academic Press.

JuLEsz, B. (1971). Foundations of Cyclopean perception. Chicago: University of Chicago Press.

LegGe, G. E. (1984). Binocular contrast summation: II. Quadratic summation. Vision Research, 24, 385-394.

LEGGE, G. E., \& FoleY, J. M. (1980). Contrast masking of human vision. Journal of the Optical Society of America, 70, 1458-1471.

MCFADDEN, D. (1968). Masking-level differences determined with and without interaural disparities in masker intensity. Journal of the Acoustical Society of America, 44, 212-223.

Moraglia, G., \& SChneider, B. (1990). Effects of direction and magnitude of horizontal disparity on binocular unmasking. Perception, $19,581-593$

Moraglia, G., \& Shneider, B. (1991). Binocular unmasking with vertical disparity. Canadian Journal of Psychology, 45, 353-366.

Moraglia, G. , Schneider, B. (1992). On binocular unmasking of signals in noise: Further tests of the summation hypothesis. Vision Research, 32, 375-385.

OGLE, K. N. (1961). Optics. Springfield, IL: Thomas.

Ohzawa, I., \& Freman, R. D. (1986a). The binocular organization of simple cells in the cat's visual cortex. Joumal of Neurophysiology, 56, 221-240.

Ohzawa, I., \& Freeman, R. D. (1986b). The binocular organization of complex cells in the cat's visual cortex. Jourmal of Neurophysiology, 56, 241-259.

Pichora-Fuller, M. K., \& Schneider, B. (1991). Masking level differences in the elderly: A comparison of antiphasic and time-delay dichotic conditions. Journal of Speech \& Hearing Research, 34, 1410-1422.

SaChS, M. B., Nachmias, J., Robson, J. G. (1971). Spatial frequency channels in human vision. Journal of the Optical Society of America, 61, 1176-1186.

Schneider, B., Moraglia, G., \& Jepson, A. (1989). Binocular unmasking: An analog to binaural unmasking? Science, 243, 1479-1481.

WAtson, A. B. (1982). Summation of grating patches indicates many types of detectors at one retinal location. Vision Research, 22, 17-25.

WOLFE, J. M. (1986). Stereopsis and binocular rivalry. Psychological Review, 93, 269-282.

\section{APPENDIX A}

\section{Vector Theory}

Figure A1 presents the vector diagrams for left and right visual fields for the condition $N_{d} S_{0}$, at the spatial frequency and orientation corresponding to that of the Gabor signal. Note that the external noise vector $\mathbf{R}_{0}$ at the signal's spatial frequency is $180^{\circ}$ out of phase in the two visual fields. The magnitude of $\mathbf{R}_{0}$, however, is the same in both fields. If $\theta_{0}$ is the phase angle of the external noise vector in the left eye, the phase angle of the external noise vector in the right eye is $\theta_{0}+180^{\circ}$. Assume that the signal is in cosine phase (as it was in this experiment). Then adding the signal to the noise vector in the two fields is equivalent to adding horizontal vector $\mathbf{s}$ to the tips of the noise vectors in both left and right visual fields. In addition to the external noise vector and signal vectors in each field, we are hypothesizing that independent random noise is added to both fields. Let $\mathbf{R}_{\mathbf{R}}$ and $\mathbf{R}_{\mathrm{L}}$ be the independent internal noise vectors added to the right and left fields, respectively. The magnitude of the resultant left-field vector is identified as $\boldsymbol{R}_{\mathbf{2}}$ in Figure A1 and its phase angle is $\theta_{2}$. Correspondingly, the resultant right-field vector has magnitude $R_{1}$, and phase angle $\theta_{1}$. The extent to which $\theta_{2}-\theta_{1}$ differs from $180^{\circ}$ can be used as a detection cue. Therefore, we are interested in the distribution of $\left(\theta_{2}-\theta_{1}\right)-180^{\circ}$. Figure A2 shows the distribution of the equivalent left- and right-field vectors for $N_{0} S_{\pi}$, and the resultant phase angles $\theta_{2}$ and $\theta_{1}$. This condition represents the case in which the left and right external noises appear in corresponding positions within the frame, and the Gabor signals also appear in corresponding positions but $180^{\circ}$ out of phase. Note that vector lengths $R_{\mathrm{R}}$ and $R_{\mathrm{L}}$, and phase angle $\theta_{1}$ are identical in both Figures $A 1$ and $A 2$ and that $\theta_{2}$ in Figure $A 2$ is shifted by $180^{\circ}$ relative to its value in Figure $A 1$. It is easy to show that when the external noise vector, signal vector, and internal noise vectors are the same 




Figure A1. Vector representation of events in the left and right eyes of an observer in the $N_{d} S_{0}$ condition. The $R_{0}$ vector in the upper right-hand quadrant is the noise vector in the right eye. The equivalent vector in the lower left-hand quadrant is the noise vector in the left eye. The vector, $s$, represents the signal added to both eyes, and $R_{R}$ and $R_{L}$ represent independent left- and right-eye noise vectors. The resultant phase angle for the right eye is labeled $\theta_{1}$; the resultant phase angle for the left eye is $\theta_{2}$. .

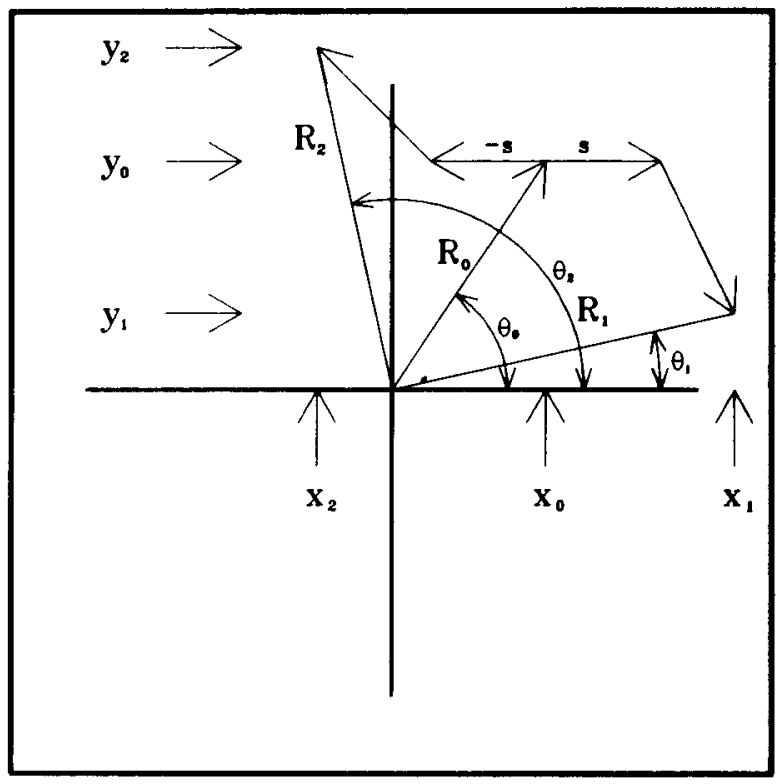

Figure A2. Vector representation of events in the left and right eyes of an observer in the $N_{0} S_{\pi}$ condition. The noise vector $R_{0}$ is common to both eyes. The vector labeled $-s$ is added to the left eye, and the vector $\mathrm{s}$ is added to the right eye. The internal noise vector added to the right eye is the same as the internal noise vector $\mathbf{R}_{\mathbf{R}}$ shown in Figure A1. The internal noise vector added to the left eye is identical in length but opposite in phase to that shown in Figure A1. The coordinates for the tip of the external noise vector are $\left(x_{0} y_{0}\right)$, the coordinates for the tip of the resultant right eye vector are $\left(x_{1}, y_{1}\right)$, and the coordinates of the resultant left eye vector are $\left(x_{2} y_{2}\right)$. The phase angles for the noise vector and the resultant right and left eye vectors are $\theta_{0}, \theta_{1}$, and $\theta_{2}$, respectively.

for $N_{d} S_{0}$ as they are for $N_{0} S_{r}$, then the distribution of $\left(\theta_{2}-\theta_{1}-180^{\circ}\right)$ in $N_{d} S_{0}$ is identical to $\theta_{2}-\theta_{1}$ in $N_{0} S_{r}$. We derive the distribution function for $\theta_{2}-\theta_{1}$ in condition $N_{0} S_{*}$, and note that it must be identical to that of $\left(\theta_{2}-\theta_{1}-180^{\circ}\right)$ in $N_{d} S_{0}$.

In Figure $\mathbf{A} 2, \mathbf{R}_{\mathbf{0}}$ is the vector corresponding to the external noise. Because we are assuming that the BMLD condition is $N_{a} S_{z}$, the external noise vector is the same in both eyes. The tip of this vector is the point, $\left(x_{0}, y_{0}\right)$, where $x_{0}=R_{0} \cos \theta_{0}$ and $y_{0}=R_{0} \sin \theta_{0}$. Let us assume, for the moment, a fixed external noise vector. Assume that the signal is added to the noise in the right eye in cosine phase. In Figure A2, the signal added to the right eye external noise is the vector $\mathbf{s}$. Because the testing conditions are $N_{0} S_{n}$, the external noise vector is the same in both eyes, but the signal vector in the left eye is opposite in phase. Thus, the left-eye signal vector is represented by $-\mathbf{s}$. We also assume that independent internal noise is added in each eye. Let us assume that the standard deviation, $\sigma_{a}$, of the internal noise in the left eye is the same as the standard deviation of the internal noise in the right eye. Adding external noise to the right eye adds a random vector, $\mathbf{R}_{\mathbf{R}}$, to the vector sum of $\mathbf{R}_{0}$ and $\mathbf{s}$. Define the tip of the resultant right-eye vector, $\mathbf{R}_{1}$, as the point $\left(x_{1}, y_{1}\right)$. Similarly, the resultant left-eye vector is the vector sum of $R_{0},-s$, and $R_{L}$. Define its tip as $\left(x_{2}, y_{2}\right)$. Clearly $x_{1}=R_{1} \cos \theta_{1}$, $y_{1}=R_{1} \sin \theta_{1}, x_{2}=R_{2} \cos \theta_{2}$, and $y_{2}=R_{2} \sin \theta_{2}$. For a given external noise vector, $\mathbf{R}_{0}$, and for $a^{2}=1 /\left(2 \sigma_{a}^{2}\right)$, the probability density function (PDF) for the point $\left(x_{1}, y_{1}\right)$ is

$$
\left(a^{2} / \pi\right) \exp \left(-a^{2} v^{2}\right) \text {, }
$$

where $v^{2}=\left[x_{1}-\left(x_{0}+s\right)\right]^{2}+\left[y_{1}-y_{0}\right]^{2}$. Therefore, the integral of this PDF is

$$
\left(a^{2} / \pi\right) \int_{-\infty}^{\infty} \int_{-\infty}^{\infty} \exp \left(-a^{2} v^{2}\right) d x_{1} d y_{1} .
$$

Now, if we switch to polar coordinates, $d x_{1} d y_{1}$ becomes $R_{1} d R_{1} d \theta_{1}$ and

$$
\begin{aligned}
v^{2} & =\left[x_{1}-\left(x_{0}+s\right)\right]^{2}+\left[y_{1}-y_{0}\right]^{2} \\
& =x_{1}^{2}-2 x_{1} x_{0}-2 x_{1} s+x_{0}^{2}+2 x_{0} s+s^{2}+y_{1}^{2}-2 y_{1} y_{0}+y_{0}^{2} \\
& =R_{1}^{2} \cos ^{2} \theta_{1}-2 R_{1} R_{0} \cos \theta_{1} \cos \theta_{0}-2 R_{1} \operatorname{scos} \theta_{1}+R_{0}^{2} \cos ^{2} \theta_{0}+s^{2}
\end{aligned}
$$




$$
\begin{aligned}
& +R_{1}^{2} \sin ^{2} \theta_{1}-2 R_{1} R_{0} \sin \theta_{1} \sin \theta_{0}+2 R_{0} s \cos \theta_{0}+R_{0}^{2} \sin ^{2} \theta_{0} \\
= & R_{1}^{2}-2 R_{2} R_{0} \cos \left(\theta_{1}-\theta_{0}\right)-2 R_{2} s \cos \theta_{1}+R_{0}^{2}+s^{2}+2 R_{0} s \cos \theta_{0} .
\end{aligned}
$$

Also, with this switch to polar coordinates, $R_{1}$ is integrated from 0 to infinity and $\theta_{1}$ is integrated from $\theta_{0}-\pi$ to $\theta_{0}+\pi$. Now define

$$
\begin{aligned}
& X=\theta_{1}-\theta_{0}, d X=d \theta_{1}, \text { with the limits of integration for } X \text { being }-\pi \text { to } \pi \text {. } \\
& \beta=R_{0} \cos X+s \cos \left(X+\theta_{0}\right) \text {. } \\
& \alpha^{2}=R_{0}^{2} \sin ^{2} X-2 R_{0} s \cos X \cos \left(X+\theta_{0}\right)+s^{2} \sin ^{2}\left(X+\theta_{0}\right)+2 R_{0} s \cos \theta_{0} \text {. }
\end{aligned}
$$

The integral of the density function now becomes

$$
\left(a^{2} / \pi\right) \int_{0}^{\infty} \int_{-\pi}^{\pi} R_{1} \exp \left[-a^{2}\left(R_{1}-\beta\right)^{2}\right] \exp \left(-a^{2} \alpha^{2}\right) d X d R_{1} .
$$

Now define $\mu=R_{1}-\beta, d \mu=d R_{1}$, with the limits of integration on $\mu$ being $-\beta$ to infinity. The integral of the density function now becomes

$$
\begin{aligned}
\left(a^{2} / \pi\right) \int_{-\pi}^{\pi} \exp \left(-a^{2} \alpha^{2}\right)\left(\int_{-\beta}^{\infty}(\mu+\beta)\right. & \left.\exp \left(-a^{2} \mu^{2}\right) d \mu\right) d X \\
& =\left(a^{2} / \pi\right) \int_{-\pi}^{\pi} \exp \left(-a^{2} \alpha^{2}\right)\left(\exp \left(-a^{2} \beta^{2}\right) /\left(2 a^{2}\right)+\beta \int_{-\beta}^{\infty} \exp \left(-a^{2} \mu^{2}\right) d \mu\right) d X .
\end{aligned}
$$

Define $t=a \mu, d t=a d \mu$, with the limits on integration for $t$ being $-a \beta$ to infinity. The integral now becomes

$$
\left(a^{2} / \pi\right) \int_{-\pi}^{\pi} \exp \left(-a^{2} \alpha^{2}\right)\left(\exp \left(-a^{2} \beta^{2}\right) /\left(2 a^{2}\right)+(\beta / a) \int_{-\alpha \beta}^{\infty} \exp \left(-t^{2}\right) d t\right) d X .
$$

Now if $\beta$ is positive, then

$$
(\beta / a) \int_{-a \beta}^{\infty} \exp \left(-t^{2}\right) d t=(\beta / a)\left(0.5 \pi^{1 / 2}+\int_{0}^{a|\beta|} \exp \left(-t^{2}\right) d t\right) .
$$

If $\beta$ is negative, then

$$
(\beta / a) \int_{-\alpha \beta}^{\infty} \exp \left(-t^{2}\right) d t=(\beta / a)\left(0.5 \pi^{1 / 2}-\int_{0}^{a|\theta|} \exp \left(-t^{2}\right) d t\right) .
$$

Therefore,

$$
(\beta / a) \int_{-a \beta}^{\infty} \exp \left(-t^{2}\right) d t=\beta \pi^{1 / 2} /(2 a)+(|\beta| / a) \int_{0}^{a|\beta|} \exp \left(-t^{2}\right) d t
$$

Now,

$$
\int_{0}^{a|\beta|} \exp \left(-t^{2}\right) d t=0.5 \pi^{1 / 2} e r f(a|\beta|)
$$

Therefore, the integral of the probability density function becomes

$$
\left(\frac{1}{2 \pi}\right) \int_{-\pi}^{x}\left\{\exp \left[-a^{2}\left(\alpha^{2}+\beta^{2}\right)\right]+a \beta \pi^{1 / 2} \exp \left(-a^{2} \alpha^{2}\right)+a|\beta| \pi^{1 / 2} \exp \left(-a^{2} \alpha^{2}\right) \operatorname{erf}(a|\beta|)\right\} d X
$$

Formula $\mathrm{A} 10$ is the integral of the probability density function for $X=\theta_{1}-\theta_{0}$. By a similar derivation, the integral of the PDF of $Y=\theta_{2}-\theta_{0}$ is

$$
\left(\frac{1}{2 \pi}\right) \int_{-\pi}^{\pi}\left\{\exp \left[-a^{2}\left(A^{2}+B^{2}\right)\right]+a B \pi^{1 / 2} \exp \left(-a^{2} A^{2}\right)+a|B| \pi^{1 / 2} \exp \left(-a^{2} A^{2}\right) \operatorname{erf}(a|B|)\right\} d Y
$$

where

$$
\begin{aligned}
& B=R_{0} \cos Y-s \cos \left(Y+\theta_{0}\right) \\
& A^{2}=R_{0}^{2} \sin ^{2} Y+2 R_{0} s \cos Y \cos \left(Y+\theta_{0}\right)+s^{2} \sin ^{2}\left(Y+\theta^{a}\right)-2 R_{0} s \cos \theta_{0} .
\end{aligned}
$$

Now the integral of the joint PDF for $X, Y$ is the product of Equations $A 10$ and A11:

$$
\begin{aligned}
& \left(\frac{1}{2 \pi}\right) \int_{-\pi}^{\pi}\left\{\exp \left[-a^{2}\left(\alpha_{1}^{2}+\beta^{2}\right)\right]+a \beta \pi^{1 / 2} \exp \left(-a^{2} \alpha_{1}^{2}\right)+a|\beta| \pi^{1 / 2} \exp \left(-a^{2} \alpha_{1}^{2}\right) \operatorname{erf}(a|\beta|)\right\} d X \\
& \times \\
& \left(\frac{1}{2 \pi}\right) \int_{-\pi}^{\pi}\left\{\exp \left[-a^{2}\left(A_{1}^{2}+B^{2}\right)\right]+a B \pi^{1 / 2} \exp \left(-a^{2} A_{1}^{2}\right)+a|B| \pi^{1 / 2} \exp \left(-a^{2} A_{1}^{2}\right) \operatorname{erf}(a|B|)\right\} d Y,
\end{aligned}
$$


where

$$
\begin{aligned}
& \alpha_{1}^{2}=R_{0}^{2} \sin ^{2} X-2 R_{0} s \cos X \cos \left(X+\theta_{0}\right)+s^{2} \sin ^{2}\left(X+\theta_{0}\right) \\
& A_{1}^{2}=R_{0}^{2} \sin ^{2} Y+2 R_{0} s \cos Y \cos \left(Y+\theta_{0}\right)+s^{2} \sin ^{2}\left(Y+\theta_{0}\right) .
\end{aligned}
$$

Now this joint PDF is defined in the $X Y$ plane. If we rotate the axes of this plane by $45^{\circ}$ to obtain axes $X_{r}, Y_{r}$, then

$$
X_{r}=\left[1 / 2^{1 / 2}\right][X+Y], Y_{r}=\left[1 / 2^{1 / 2}\right][Y-X],
$$

and

$$
X=\left[1 / 2^{1 / 2}\right]\left[X_{r}-Y_{r}\right], Y=\left[1 / 2^{1 / 2}\right]\left[Y_{r}+X_{r}\right] \text {. }
$$

In the $X_{r}, Y_{r}$ plane, the limits of integration for $Y_{r}$ are $-2^{1 / 2} \pi$ to $2^{1 / 2} \pi$. Let $c=2^{1 / 2}$. The limits of integration for $X_{r}$ are from $-L$ to $+L$, where

$$
L=\left|\left[\left|Y_{r}\right|-c \pi\right]\right| .
$$

Therefore, the integral of the probability density function in the $X_{r}, Y_{r}$ plane is

$$
\left(\frac{1}{4 \pi^{2}}\right) \int_{-c \pi}^{c \pi} \int_{-L}^{L}\left(\begin{array}{c}
\exp \left[-a^{2}\left(R_{0}^{2}+s^{2}\right)\right]+a \beta_{2} \pi^{1 / 2} \exp \left(-a^{2} \alpha_{2}^{2}\right)+a\left|\beta_{2}\right| \pi^{1 / 2} \exp \left(-a^{2} \alpha_{2}^{2}\right) \operatorname{erf}\left(a\left|\beta_{2}\right|\right) \\
\times \\
\exp \left[-a^{2}\left(R_{0}^{2}+s^{2}\right)\right]+a B_{2} \pi^{1 / 2} \exp \left(-a^{2} A_{2}^{2}\right)+a\left|B_{2}\right| \pi^{1 / 2} \exp \left(-a^{2} A_{2}^{2}\right) \operatorname{erf}\left(a\left|B_{2}\right|\right)
\end{array}\right) d X_{r} d Y_{r}
$$

where,

$$
\begin{aligned}
\beta_{2} & =R_{0} \cos \left[\left(X_{r}-Y_{r}\right) / c\right]+s \cos \left[\left(X_{r}-Y_{r}\right) / c+\theta_{0}\right] \\
B_{2} & =R_{0} \cos \left[\left(Y_{r}+X_{r}\right) / c\right]-s \cos \left[\left(Y_{r}+X_{r}\right) / c+\theta_{0}\right] \\
\alpha_{2}^{2} & =R_{0}^{2} \sin ^{2}\left[\left(X_{r}-Y_{r}\right) / c\right]-2 R_{0} s \cos \left[\left(X_{r}-Y_{r}\right) / c\right] \cos \left[\left(X_{r}-Y_{r}\right) / c+\theta_{0}\right] \\
& +s^{2} \sin ^{2}\left[\left(X_{r}-Y_{r}\right) / c+\theta_{0}\right] \\
A_{2}^{2} & =R_{0}^{2} \sin ^{2}\left[\left(Y_{r}+X_{r}\right) / c\right]+2 R_{0} \operatorname{scos}\left[\left(Y_{r}+X_{r}\right) / c\right] \cos \left[\left(Y_{r}+X_{r}\right) / c+\theta_{0}\right] \\
& +s^{2} \sin ^{2}\left[\left(Y_{r}+X_{r}\right) / c+\theta_{0}\right] .
\end{aligned}
$$

Now, with a change of variables, we can define

$$
\begin{aligned}
& \theta_{d}=c Y_{r}=\theta_{2}-\theta_{1} \\
& \theta_{p}=c X_{r}=\theta_{1}+\theta_{2}-2 \theta_{0} .
\end{aligned}
$$

The integral then becomes

$$
\left(\frac{1}{8 \pi^{2}}\right) \int_{-2 \pi}^{2 \pi} \int_{-L p}^{L p}\left(\begin{array}{c}
\exp \left[-a^{2}\left(R_{0}^{2}+s^{2}\right)\right]+a \beta_{d} \pi^{1 / 2} \exp \left(-a^{2} \alpha_{d}^{2}\right)+a\left|\beta_{d}\right| \pi^{1 / 2} \exp \left(-a^{2} \alpha_{a}^{2}\right) \operatorname{erf}\left(a\left|\beta_{d}\right|\right) \\
\times \\
\exp \left[-a^{2}\left(R_{0}^{2}+s^{2}\right)\right]+a B_{p} \pi^{1 / 2} \exp \left(-a^{2} A_{p}^{2}\right)+a\left|B_{p}\right| \pi^{1 / 2} \exp \left(-a^{2} A_{p}^{2}\right) \operatorname{erf}\left(a\left|B_{p}\right|\right)
\end{array}\right) d \theta_{p} d \theta_{d}
$$

where

$$
\begin{aligned}
& L_{p}=\left|\left(\left|\theta_{d}\right|-2 \pi\right)\right| \\
& \beta_{d}=R_{0} \cos \left[.5\left(\theta_{p}-\theta_{d}\right)\right]+s \cos \left[.5\left(\theta_{p}-\theta_{d}\right)+\theta_{0}\right] \\
& B_{p}=R_{0} \cos \left[.5\left(\theta_{p}+\theta_{d}\right)\right]-s \cos \left[.5\left(\theta_{p}+\theta_{d}\right)+\theta_{0}\right] \\
& \alpha_{d}^{2}=R_{0}^{2} \sin ^{2}\left[.5\left(\theta_{p}-\theta_{d}\right)\right]-2 R_{0} s \cos \left[.5\left(\theta_{p}-\theta_{d}\right)\right] \cos \left[.5\left(\theta_{p}-\theta_{d}\right)+\theta_{0}\right]+s^{2} \sin ^{2}\left[.5\left(\theta_{p}-\theta_{d}\right)+\theta_{0}\right] \\
& A_{p}^{2}=R_{0}^{2} \sin ^{2}\left[.5\left(\theta_{p}+\theta_{d}\right)\right]+2 R_{0} \operatorname{scos}\left[.5\left(\theta_{p}+\theta_{d}\right)\right] \cos \left[.5\left(\theta_{p}+\theta_{d}\right)+\theta_{0}\right]+s^{2} \sin ^{2}\left[.5\left(\theta_{p}+\theta_{d}\right)+\theta_{0}\right] .
\end{aligned}
$$

Note that Formula A14 is conditional on $R_{0}, \theta_{0}$. Therefore, if we multiply Formula A14 by the PDF for $R_{0}, \theta_{0}$, which is $\left[R_{0} /\left(2 \sigma_{b}^{2}\right)\right] \exp \left[-R_{0}^{2} /\left(2 \sigma_{b}^{2}\right)\right] d R_{0} d \theta_{0}$, and integrate over $\theta_{0}$ and $R_{0}$, we obtain the integral of the PDF for $\theta_{d}$. Letting $b^{2}=1 /\left(2 \sigma_{b}^{2}\right)$, where $\sigma_{b}$ is the standard deviation of the external noise, this integral is

$$
\left(\frac{b^{2}}{8 \pi^{3}}\right) \int_{-2 \pi}^{2 \pi} \int_{-\pi}^{\pi} \int_{0}^{\infty} \int_{-L p}^{L p} R_{0} f\left(a, R_{0}, \theta_{0}, \theta_{p}, \theta_{d}, s\right) \exp \left(-b^{2} R_{0}^{2}\right) d \theta_{p} d R_{0} d \theta_{0} d \theta_{d}
$$


where $f\left(a, R_{0} \theta_{0}, \theta_{p}, \theta_{d}, s\right)=$

$$
\left(\begin{array}{c}
\exp \left[-a^{2}\left(R_{0}^{2}+s^{2}\right)\right]+a \beta_{d} \pi^{1 / 2} \exp \left(-a^{2} \alpha_{d}^{2}\right)+a\left|\beta_{d}\right| \pi^{1 / 2} \exp \left(-a^{2} \alpha_{d}^{2}\right) \operatorname{erf}\left(a\left|\beta_{d}\right|\right) \\
\times \\
\exp \left[-a^{2}\left(R_{0}^{2}+s^{2}\right)\right]+a B_{p} \pi^{1 / 2} \exp \left(-a^{2} A_{p}^{2}\right)+a\left|B_{p}\right| \pi^{1 / 2} \exp \left(-a^{2} A_{p}^{2}\right) \operatorname{erf}\left(a\left|B_{p}\right|\right)
\end{array}\right)
$$

Therefore, the PDF for $\theta_{d}$ is

$$
\left(\frac{b^{2}}{8 \pi^{3}}\right) \int_{-\pi}^{\pi} \int_{0}^{\infty} \int_{-L p}^{L_{p}} R_{0} f\left(a, R_{0}, \theta_{0}, \theta_{p}, \theta_{d}, s\right) \exp \left(-b^{2} R_{0}^{2}\right) d \theta_{p} d R_{0} d \theta_{0},
$$

where $\theta_{d}$ ranges from $-2 \pi$ to $2 \pi$.

We can now ask what happens if we attenuate both the noise and the signal vectors in the left eye by scale factor $r$. This has the effect of multiplying both $R_{0}$ and $s$ in the left eye by $r$. This in turn requires that $B$ be multiplied by $r$ and that $A^{2}$ be multiplied by $r^{2}$. If we follow through on these changes, we can show that the probability density function for $\theta_{d}$ is

$$
\left(\frac{b^{2}}{8 \pi^{3}}\right) \int_{-\pi}^{\pi} \int_{0}^{\infty} \int_{-L p}^{L_{p}} \exp \left[-2 a^{2} R_{0} s \cos \theta_{0}\left(1-r^{2}\right)\right] R_{0} g\left(a, R_{0}, \theta_{0}, \theta_{p}, \theta_{d}, s, r\right) \exp \left(-b^{2} R_{0}^{2}\right) d \theta_{p} d R_{0} d \theta_{0},
$$

where $g\left(a, R_{0}, \theta_{0}, \theta_{p}, \theta_{d}, s, r\right)=$

$$
\left(\begin{array}{c}
\exp \left[-a^{2}\left(R_{0}^{2}+s^{2}\right)\right]+a \beta_{d} \pi^{1 / 2} \exp \left(-a^{2} \alpha_{d}^{2}\right)+a\left|\beta_{d}\right| \pi^{1 / 2} \exp \left(-a^{2} \alpha_{d}^{2}\right) e r f\left(a\left|\beta_{d}\right|\right) \\
\times \\
\exp \left[-a^{2} r^{2}\left(R_{0}^{2}+s^{2}\right)\right]+a r B_{p} \pi^{1 / 2} \exp \left(-a^{2} r^{2} A_{p}^{2}\right)+a\left|r B_{p}\right| \pi^{1 / 2} \exp \left(-a^{2} r^{2} A_{p}^{2}\right) \operatorname{erf}\left(a\left|r B_{p}\right|\right)
\end{array}\right)
$$

Equation A18 was used to generate the probability density functions shown in Figure 9. To evaluate the error function (erf), approximation 7.1.28 in Abramowitz and Stegun (1970) was employed. The three integrals in Equation A18 were evaluated numerically. Note that $\theta_{d}$ is evaluated from $-2 \pi$ to $2 \pi$. Since $\theta_{d}$ $=+190^{\circ}$ is the same angle as $\theta_{d}=-170^{\circ}$, the functions in Figure 9 are plotted between $-\pi$ and $\pi$, taking into account the equivalence noted above.

\section{APPENDIX B \\ The Summation Model}

To show that the summation model provides a good fit to the data, let $g(x, y)$ have the spectral density function given in Formula 2. Similarly, let the density function for the internal Gaussian noise $h(x, y)$ be

$$
\begin{aligned}
H[\epsilon, \eta]= & A_{h},-\epsilon_{u}<\epsilon<\epsilon_{u} \text { and }-\eta_{u}<\eta<\eta_{u} \\
& 0, \text { elsewhere, }
\end{aligned}
$$

where $\epsilon_{u}, \eta_{u}$ are the horizontal and vertical frequency band limits on the internal Gaussian noise. For the purpose of evaluating BMLDs, let us assume that $\epsilon_{u}$ and $\eta_{u}$ are effectively equal to infinity. This assumption is reasonable provided that the bandwidth of the internal Gaussian filter is small relative to the bandwidths of the external and internal noises. Let $d=d_{x}+d_{\alpha}$ be the combined external and internal horizontal shifts in the external noise. Then the spectral density function for

$$
r_{\mathrm{L}} g(x, y)+r_{\mathrm{R}} g(x+d, y)+h_{\mathrm{R}}(x, y)+h_{\mathrm{L}}(x, y),
$$

assuming that $h_{\mathrm{L}}(x, y)$ and $h_{\mathrm{R}}(x, y)$ are independent of each other and with $r_{\mathrm{L}}, r_{\mathrm{R}}$ being attenuation factors placed on the left- and right-eye external noises, becomes

$$
G(\epsilon, \eta)\left[r_{\mathrm{L}}^{2}+r_{\mathrm{R}}^{2}+2 r_{\mathrm{L}} r_{\mathrm{R}} \cos (2 \pi \epsilon d)\right]+2 H[\epsilon, \eta] .
$$

Assume now that both internal and external noises are passed through an internal Gaussian filter whose spectral profile is

$$
\exp \left[-b^{2}\left(\epsilon-\epsilon_{s}\right)^{2}-b^{2} \eta^{2}\right],
$$

where $\epsilon_{s}$ is the center frequency of the Gabor signal. Then the total energy from both internal and external noise sources in this channel is given by 


$$
\begin{aligned}
\left(G(\epsilon, \eta)\left[r_{\mathbf{L}}^{2}+r_{\mathrm{R}}^{2}\right]+2 H(\epsilon, \eta)\right) \int_{-\infty}^{\infty} & \int_{-\infty}^{\infty} \exp \left[-b^{2}\left(\epsilon-\epsilon_{s}\right)^{2}-b^{2} \eta^{2}\right] d \epsilon d \eta \\
& +\left(2 r_{\mathrm{L}} r_{\mathrm{R}} G(\epsilon, \eta)\right) \int_{-\infty}^{\infty} \int_{-\infty}^{\infty} \cos (2 \pi \epsilon d) \exp \left[-b^{2}\left(\epsilon-\epsilon_{s}\right)^{2}-b^{2} \eta^{2}\right] d \epsilon d \eta .
\end{aligned}
$$

It is relatively easy to show that this integral becomes

$$
\frac{\pi}{b^{2}}\left[G(\epsilon, \eta)\left(r_{\mathrm{L}}^{2}+r_{\mathrm{R}}^{2}\right)+2 H(\epsilon, \eta)+2 r_{\mathrm{L}} r_{\mathrm{R}} G(\epsilon, \eta) \cos \left(2 \pi d \epsilon_{s}\right) \exp \left(\frac{-\pi^{2} d^{2}}{b^{2}}\right)\right]
$$

Note that in $N_{d} S_{0}$ no shift is imposed on the Gabor signal. Therefore, maximum energy in the Gabor signal occurs when the internal horizontal shift, $d_{\alpha}=0$. Note also that the energy in the background noise is at a minimum for $d=d_{x}$, and $\epsilon_{s}=1 /\left(2 d_{x}\right)$. For in that case, the total energy in the background noise is given by

$$
\frac{\pi}{b^{2}}\left[G(\epsilon, \eta)\left(r_{\mathrm{L}}^{2}+r_{\mathrm{R}}^{2}\right)+2 H(\epsilon, \eta)-2 r_{\mathrm{L}} r_{\mathrm{R}} G(\epsilon, \eta) \exp \left(\frac{-\pi^{2} d^{2}}{b^{2}}\right)\right] .
$$

In $N_{d} S_{d}$, the Gabor is shifted by the same amount as the noise. Therefore, total energy in the summated signal is maximized when $d_{\alpha}=-d_{x}$. Hence, $d=0$, and the total energy in the background noise is given by

$$
\frac{\pi}{b^{2}}\left[G(\epsilon, \eta)\left(r_{\mathrm{L}}+r_{\mathrm{R}}\right)^{2}+2 H(\epsilon, \eta)\right]
$$

Note that for both $N_{d} S_{0}\left(d_{\alpha}=0\right)$ and $N_{d} S_{d}\left(d_{\alpha}=-d_{x}\right)$, the summated Gabor signals are identical and, therefore, the energy in summated Gabor signals is identical. The signal-to-noise ratio in $N_{d} S_{0}$ divided by the signal-tonoise ratio in $N_{d} S_{d}$ for equal-strength Gabor signals is

$$
\frac{\left[G(\epsilon, \eta)\left(r_{\mathrm{L}}+r_{\mathrm{R}}\right)^{2}+2 H(\epsilon, \eta)\right]}{\left[G(\epsilon, \eta)\left(r_{\mathrm{L}}^{2}+r_{\mathrm{R}}^{2}\right)+2 H(\epsilon, \eta)-2 r_{\mathrm{L}} r_{\mathrm{R}} G(\epsilon, \eta) \exp \left(\frac{-\pi^{2} d^{2}}{b^{2}}\right)\right]}
$$

The predicted size of the BMLD then is

$$
10 \log \left(\frac{\left[G(\epsilon, \eta)\left(r_{\mathrm{L}}+r_{\mathrm{R}}\right)^{2}+2 H(\epsilon, \eta)\right]}{\left(G(\epsilon, \eta)\left(r_{\mathrm{L}}^{2}+r_{\mathrm{R}}^{2}\right)+2 H(\epsilon, \eta)-2 r_{\mathrm{L}} r_{\mathrm{R}} G(\epsilon, \eta) \exp \left[\frac{-\pi^{2} d^{2}}{b^{2}}\right]\right)}\right]
$$

Note that because we are dealing in power rather than luminance, decibels are defined as 10 times the logarithm of the ratio of two powers rather than 20 times the logarithm of the ratio of two luminances. Now if we define $c=H(\epsilon, \eta) / G(\epsilon, \eta)$, then Equation B8 becomes

$$
\operatorname{lol}\left(\frac{\left[\left(r_{L}+r_{R}\right)^{2}+2 c\right]}{\left(\left(r_{L}^{2}+r_{R}^{2}\right)+2 c-2 r_{L} r_{R} \exp \left[\frac{-\pi^{2} d^{2}}{b^{2}}\right]\right)}\right] .
$$

Values of $b$ and $c$ were found that minimized the sum of squared deviations between predicted (Formula B9) and obtained BMLDs for all 3 subjects using an iterative procedure. The smooth curves in Figure 12 for the equal conditions were generated by forcing $r_{L}=r_{R}$ and letting $r_{R}$ vary from .22 to 1 . The smooth curves in Figure 12 for the unequal conditions were generated by forcing $r_{\mathrm{L}}=1$ and letting $r_{\mathrm{R}}$ vary from .2 to 1 .

In the equal conditions, $r_{\mathrm{L}}=r_{\mathrm{R}}=r$. In the unequal conditions, $r_{\mathrm{L}}=1$, and $r_{\mathrm{R}}=r$. Therefore, the BMLD for a reduction in one eye only will equal the BMLD for the equivalent reduction in both eyes, only when

$$
\frac{(1+r)^{2}+2 c}{1+r^{2}+2 c-2 r\left[\exp \left(\frac{-\pi^{2} d^{2}}{b^{2}}\right)\right]}=\frac{4 r^{2}+2 c}{2 r^{2}+2 c-2 r^{2}\left[\exp \left(\frac{-\pi^{2} d^{2}}{b^{2}}\right)\right]} \text {. }
$$

It can be easily verified that this equality holds when $c=0.5 r(1-r)$. For $c=0.5 r(1-r)+\epsilon, \epsilon \neq 0$, we can also show that Equation B10 is true only when

$$
\epsilon\left[1+\exp \left(\frac{-\pi^{2} d^{2}}{b^{2}}\right)\right]=\epsilon r\left[1+\exp \left(\frac{-\pi^{2} d^{2}}{b^{2}}\right)\right] .
$$


This can occur only for $\epsilon=0$, or $r=1$. Therefore, the BMLD for a reduction in one eye will equal the BMLD for the equivalent reduction in both eyes if and only if $c=0.5 r(1-r)$. For $c<0.5 r(1-r)$, the BMLD for the equal-reduction case will always be greater than the BMLD for the equivalent reduction in one eye only. Because the smallest value that $r$ takes on in this experiment is 0.25 , as long as $c<3 / 32$, the ordinal prediction in the summation model is opposite that for the vector model. Estimates of $c$ in this experiment are considerably less than this value so that the summation model predicts the opposite pattern from that of the vector model.

When there is no external noise, the signal-to-noise ratio in the left channel is $S_{\mathrm{L}} / \sigma_{\mathrm{L}}$, where $S_{\mathrm{L}}$ is the RMS amplitude of the signal in the left eye, and $\sigma_{\mathrm{L}}$ is the standard deviation of the internal noise in the left channel. Let us assume that the observer has independent access to the two monocular channels and to the binocular channel. Therefore, when the signal is presented only to the left eye, threshold will depend only on the signalto-noise ratio in the left-eye channel. Let $S_{\mathrm{LT}}$ be the RMS of the signal at threshold for monocular, left-eye presentation. The signal-to-noise ratio required for threshold in the left-eye monocular channel is, therefore, $S_{\mathrm{LT}} / \sigma_{\mathrm{L}}$. Let us furthermore assume that the signal-to-noise ratio required for threshold is the same in the binocular channel and the monocular right-eye channel. Therefore, $S_{\mathrm{LT}} / \sigma_{\mathrm{L}}=S_{\mathrm{RT}} / \sigma_{\mathrm{R}}$, where $S_{\mathrm{RT}}$ is the monocular threshold in the right eye, and $\sigma_{\mathrm{R}}$ is the right-eye internal noise. We note that sometimes the right- and left-eye thresholds are not equal, that is, $S_{\mathrm{LT}}=a S_{\mathrm{RT}}$. When this occurs, then $\sigma_{\mathrm{L}}=a \sigma_{\mathrm{R}}$, if the signal-to-noise ratio at threshold is to be identical in both monocular channels.

Now consider what happens in the binocular channel when the left-eye signal is presented at full value, $S$, with the right-eye signal set to $r S$. If the left and right internal noises are independent, the signal-to-noise ratio in the binocular channel is given by

$$
\frac{S+r S}{\sqrt{\sigma_{\mathrm{L}}^{2}+\sigma_{\mathrm{R}}^{2}}}=\frac{S(1+r)}{\sigma_{\mathrm{R}} \sqrt{1+a^{2}}} .
$$

We can now look for the value of $S$ that will produce the same signal-to-noise ratio in the binocular channel as that produced in the monocular channel when the monocular channel is at threshold. Setting the expression $\mathrm{B} 12=S_{\mathrm{RT}} / \sigma_{\mathrm{R}}$, we find the value of $S$ at binocular threshold to be

$$
S_{\mathrm{BT}}=\frac{\sqrt{S_{\mathrm{RT}}^{2}+S_{\mathrm{LT}}^{2}}}{1+r},
$$

where $S_{\mathrm{BT}}$ is the threshold value of $S$ in the binocular channel. If the observer has independent access to each of these three channels, then threshold will be reached whenever $S_{\mathrm{R}}>S_{\mathrm{RT}}$, or $S_{\mathrm{L}}>S_{\mathrm{LT}}$, or $S>S_{\mathrm{BT}}$. This is the model that was used to plot the predicted values in Figure 13.

(Manuscript received July 25, 1991; revision accepted for publication May 19, 1992.) 